\title{
Coordinated Beamforming With Relaxed Zero Forcing: The Sequential Orthogonal Projection Combining Method and Rate Control
}

\author{
Juho Park, Student Member, IEEE, Gilwon Lee, Student Member, IEEE, Youngchul Sung, Senior Member, IEEE, \\ and Masahiro Yukawa, Member, IEEE
}

\begin{abstract}
In this paper, coordinated beamforming based on relaxed zero forcing (RZF) for $K$ transmitter-receiver pair multiple-input single-output (MISO) and multiple-input multiple-output (MIMO) interference channels is considered. In the RZF coordinated beamforming, conventional zero-forcing interference leakage constraints are relaxed so that some predetermined interference leakage to undesired receivers is allowed in order to increase the beam design space for larger rates than those of the zero-forcing ( $\mathrm{ZF}$ ) scheme or to make beam design feasible when ZF is impossible. In the MISO case, it is shown that the rate-maximizing beam vector under the RZF framework for a given set of interference leakage levels can be obtained by sequential orthogonal projection combining (SOPC). Based on this, exact and approximate closed-form solutions are provided in two-user and three-user cases, respectively, and an efficient beam design algorithm for RZF coordinated beamforming is provided in general cases. Furthermore, the rate control problem under the RZF framework is considered. A centralized approach and a distributed heuristic approach are proposed to control the position of the designed rate-tuple in the achievable rate region. Finally, the RZF framework is extended to MIMO interference channels by deriving a new lower bound on the rate of each user.
\end{abstract}

Index Terms-Coordinated beamforming, inter-cell interference, multi-cell MIMO, Pareto-optimal, rate control, relaxed zero-forcing, sequential orthogonal projection combining.

\section{INTRODUCTION}

$\mathbf{I}$ $\mathrm{N}$ current and future cellular networks, handling interference in the network is one of the most critical problems. Among the many ways of handling interference, MIMO antenna

Manuscript received September 26, 2012; revised February 05, 2013; accepted April 05, 2013. Date of publication April 16, 2013; date of current version May 20, 2013. The associate editor coordinating the review of this manuscript and approving it for publication was Prof. Martin Haardt. This research was supported by the Basic Science Research Program through the National Research Foundation of Korea (NRF) funded by the Ministry of Education, Science and Technology (2010-0021269). Part of this paper was presented at the International Conference on Wireless Communications and Signal Processing (WCSP), Nanjing, China, November 2011 [1].

J. Park, G. Lee, and Y. Sung are with the Department of Electrical Engineering, KAIST, Daejeon, South Korea, 305-701 (e-mail: jhp@ee.kaist.ac.kr; gwlee@ee.kaist.ac.kr; ysung@ee.kaist.ac.kr).

M. Yukawa is with the Department of Electronics and Electrical Engineering, Keio University, Yokohama, Kanagawa, 223-8522, Japan (e-mail: yukawa@elec.keio.ac.jp).

This paper has supplementary downloadable multimedia material available at http://ieeexplore.ieee.org provided by the authors. This includes a rar file, multimedia.rar, which contains Matlab source codes for the figures in the paper. Please read the readme file in each subdirectory. This material is 406KB in size.

Color versions of one or more of the figures in this paper are available online at http://ieeexplore.ieee.org.

Digital Object Identifier 10.1109/TSP.2013.2258343 techniques and base station cooperation are considered as the key technologies to the interference problem. Indeed, the 3GPP Long-Term Evolution-Advanced considers the base station cooperation and MIMO techniques to mitigate inter-cell interference under the name of Coordinated Multipoint (CoMP) [2], [3] . Mathematically, when each mobile station has a single receive antenna and data is not shared among base stations, the system is modelled as a MISO interference channel (IC), and extensive research has been conducted on beam design for this MISO IC, especially under the assumption of practical linear beamforming treating interference as noise. First, Jorswieck et al. investigated the structure of optimal beam vectors achieving Pareto boundary points of the achievable rate region of the MISO IC with linear beamforming [4] and showed that any Pareto-optimal beam vector at each transmitter is a normalized convex combination of the ZF beam vector and matched-filtering (MF) (i.e., maximal ratio transmission) beam vector in the case of two users and a linear combination of the channel vectors from the transmitter to all receivers in the general case of an arbitrary number of users. The result is extended in [5] to general MISO interference networks with arbitrary utility functions having monotonic property. Moreover, the parameterization for the Pareto-optimal beam vector is compressed from $K(K-$ 1) complex numbers [4] to $K(K-1)$ real numbers. In addition to these results, other interesting works for MISO ICs include the consideration of imperfect CSI [6], shared data [7], second-order cone programming [8], monotonic optimization with polyblock algorithm [9], etc. Although these works provide significant theoretical insights into the optimal beam structure and parameterization of Pareto-optimal beam vectors, it is not easy to use these results to design an optimal beam vector in the real-world systems, and the beam design problem in the general case still remains as a non-trivial problem practically.

With a sufficient number of transmit antennas, the simplest beam design method for base station coordination is ZF, which perfectly eliminates interference leakage to undesired receivers. However, it is well known that the ZF method is not optimal in the sense of sum data rate or Pareto-boundary achievability, and there have been several ideas to enhance the ZF beam design method. In the case of multi-user MISO/MIMO broadcast channels, the regularized channel inversion (RCI) [10]-[12] and the signal-to-leakage-plus-noise ratio (SLNR) method [13] were proposed for this purpose. In particular, the SLNR method maximizes the ratio of signal power (to the desired receiver) to leakage (to undesired receivers) plus noise power, and its solution is given by solving a generalized eigenvalue problem. The SLNR method can easily be adapted to the MISO/MIMO IC. 
Recently, Zakhour and Gesbert rediscovered this method in the context of MISO IC under the name of the virtual signal-tointerference-plus-noise ratio (SINR) method, and have further (and more importantly) shown that this method can achieve any point on the Pareto boundary theoretically, but practically can achieve one uncontrolled point on the Pareto boundary of the achievable rate region in the case of two ${ }^{1}$ users [15], [16].

Another way of generalizing ZF in MISO IC was proposed by relaxing the ZF leakage constraints to undesired users in [17], [18], [1]. First, Shang et al. showed that all boundary points of the achievable rate region of MISO IC with single-user decoding can be obtained by linear beamforming [17], by converting the non-convex weighted sum rate maximizing precoder design problem into a set of separate convex problems by taking a lower bound on the achievable rate of each user under the relaxed ZF (RZF) framework. This method was further investigated by Zhang and Cui [18], who showed that separate rate optimization under the RZF framework with a set of well-chosen interference leakage levels to undesired users is Pareto-optimal for MISO ICs in addition to being sum-rate optimal. In [1], Lee et al. extended the RZF framework to the case of MIMO IC. In this RZF beamforming framework, each transmitter maximizes its own rate under interference leakage constraints to undesired receivers. The idea is based on the simple observation that the ZF beam design method overreacts to inter-cell interference by completely nulling out the interference. Most receivers (i.e., mobile stations) that are affected by inter-cell interference are cell-edge users, and thus, thermal noise remains even if the inter-cell interference is completely removed. Thus, it is unnecessary to completely eliminate the inter-cell interference and it is sufficient to limit the inter-cell interference to a certain level comparable to that of the thermal noise. By relaxing ZF interference constraints, we do not need the condition that the number of transmit antenna is larger than or equal to that of receivers and have a larger feasible set yielding a larger rate than that of the ZF scheme. In this paper, we explore and develop this RZF idea fully in several aspects to provide a useful design paradigm for coordinated beamforming (CB) for current and future cellular networks. The contributions of the paper is summarized as follows:

- In the MISO IC case, a new structural representation of optimal beam vector for RZF coordinate beamforming is derived.

- In the MISO IC case, based on the new structural representation, the sequential orthogonal projection combining (SOPC) method for the RZF beam design is proposed.

- In the RZF framework, the allowed interference leakage levels to undesired receivers at each transmitter are design parameters, and the rate-tuple is controlled by controlling these interference leakage levels. A centralized algorithm and a fully distributed heuristic algorithm are provided to control the location of the designed rate-tuple (roughly) along the Pareto boundary of the achievable rate region. The controllability of rate is a desirable feature in network operation since the required data rate of each transmitter-receiver pair may be different from those of others

\footnotetext{
${ }^{1}$ It can be shown that the virtual SINR (or SLNR) method can theoretically achieve any Pareto-optimal point in the general MISO IC case, too. See the Appendix of [14].
}

in practice, as in an example that one user is a voice user and the others are high rate data users.

- Finally, the RZF CB (RZFCB) is extended to the MIMO IC case. In the MIMO case, a new lower bound on each user's rate is derived to decompose the beam design problem into separate problems at different transmitters, and the projected gradient method [19] is adopted to solve the MIMO RZFCB problem.

Notations and Organization: In this paper, we will make use of standard notational conventions. Vectors and matrices are written in boldface with matrices in capitals. All vectors are column vectors. For a matrix $\mathbf{A}, \mathbf{A}^{H},\|\mathbf{A}\|,\|\mathbf{A}\|_{F}, \operatorname{tr}(\mathbf{A})$, and $|\mathbf{A}|$ indicate the Hermitian transpose, 2-norm, Frobenius norm, trace, and determinant of $\mathbf{A}$, respectively, and $\mathcal{C}(\mathbf{A})$ denotes the column space of $\mathbf{A}$. $\mathbf{I}_{n}$ stands for the identity matrix of size $n$ (the subscript is omitted when unnecessary). $\boldsymbol{\Pi}_{\mathbf{A}}=\mathbf{A}\left(\mathbf{A}^{H} \mathbf{A}\right)^{-1} \mathbf{A}^{H}$ represents the orthogonal projection ${ }^{2}$ onto $\mathcal{C}(\mathbf{A})$ and $\boldsymbol{\Pi}_{\mathbf{A}}^{\perp}=\mathbf{I}-\mathbf{\Pi}_{\mathbf{A}}$. For matrices $\mathbf{A}$ and $\mathbf{B}, \mathbf{A} \geq \mathbf{B}$ means that $\mathbf{A}-\mathbf{B}$ is positive semi-definite. $\left[\mathbf{a}_{1}, \ldots, \mathbf{a}_{L}\right]$ or $\left[\mathbf{a}_{i}\right]_{i=1}^{L}$ denotes the matrix composed of vectors $\mathbf{a}_{1}, \ldots, \mathbf{a}_{L}$. $\mathbf{x} \sim \mathcal{C N}(\boldsymbol{\mu}, \boldsymbol{\Sigma})$ means that $\mathbf{x}$ is circular-symmetric complex Gaussian-distributed with mean vector $\boldsymbol{\mu}$ and covariance matrix $\Sigma . \mathbb{R}, \mathbb{R}_{+}$, and $\mathbb{C}$ denote the sets of real numbers, non-negative real numbers, and complex numbers, respectively. For a set $A$, $|A|$ represents the cardinality of the set.

The remainder of this paper is organized as follows. The system model and the preliminaries are provided in Section II. In Section III, the RZFCB in MISO ICs is formulated, and its solution structure and a fast algorithm for RZFCB are provided. In Section IV, the rate-tuple control problem under the RZFCB framework is considered and two approaches are proposed to control the designed rate-tuple. The RZFCB problem in MIMO ICs is considered in Section V, followed by conclusions in Section VI.

\section{System Model ANd Preliminaries}

In this paper, we consider a multi-user interference channel with $K$ transmitter-receiver pairs. In the first part of the paper, we restrict ourselves to the case that the transmitters are equipped with $N$ antennas and each receiver is equipped with one receive antenna only. In this case, the received signal at receiver $i$ is given by

$$
y_{i}=\mathbf{h}_{i i}^{H} \mathbf{v}_{i} s_{i}+\sum_{j=1, j \neq i}^{K} \mathbf{h}_{i j}^{H} \mathbf{v}_{j} s_{j}+n_{i},
$$

where $\mathbf{h}_{i j}$ denotes the $N \times 1$ (conjugated) channel vector from transmitter $j$ to receiver $i$, and $\mathbf{v}_{j}$ and $s_{j}$ are the $N \times 1$ beamforming vector and the scalar transmit symbol at transmitter $j$, respectively. We assume that the transmit symbols are from a Gaussian code book with unit variance, the additive noise $n_{i}$ is from $\mathcal{C N}\left(0, \sigma_{i}^{2}\right)$, and each transmitter has a transmit power constraint, $\left\|\mathbf{v}_{i}\right\|^{2} \leq P_{i}, i=1, \ldots, K$.

The first term on the right-hand side (RHS) of (1) is the desired signal and the second term represents the sum of interference from $K-1$ undesired transmitters. Under single-user decoding at each receiver treating interference as noise, for a

\footnotetext{
${ }^{2}$ In general, the projection is given with a pseudoinverse of $\mathbf{A}^{H}$.
} 
given set of beamforming vectors $\left\{\mathbf{v}_{1}, \ldots, \mathbf{v}_{K}\right\}$ and a channel realization $\left\{\mathbf{h}_{i j}\right\}$, the rate of receiver $i$ is given by

$$
R_{i}\left(\mathbf{v}_{1}, \ldots, \mathbf{v}_{K}\right)=\log _{2}\left(1+\frac{\left|\mathbf{h}_{i i}^{H} \mathbf{v}_{i}\right|^{2}}{\sigma_{i}^{2}+\sum_{j \neq i}\left|\mathbf{h}_{i j}^{H} \mathbf{v}_{j}\right|^{2}}\right) .
$$

Then, for the given channel realization, the achievable rate region of the MISO IC with transmit beamforming and single-user decoding is defined as the union of the rate-tuples that can be achieved by all possible combinations of beamforming vectors under the power constraints:

$$
\begin{aligned}
& \mathcal{R}:=\quad \bigcup \quad\left(R_{1}\left(\mathbf{v}_{1}, \ldots, \mathbf{v}_{K}\right), \ldots,\right. \\
& \left\{\begin{array}{c}
\mathbf{v}_{i}: \mathbf{v}_{i} \in \mathbb{C}^{N} \\
\left\|\mathbf{v}_{i}\right\|^{2} \leq P_{i}, 1 \leq i \leq K
\end{array}\right\} \\
& \left.R_{K}\left(\mathbf{v}_{1}, \ldots, \mathbf{v}_{K}\right)\right) .
\end{aligned}
$$

The outer boundary of the rate region $\mathcal{R}$ is called the Pareto boundary of $\mathcal{R}$ and it consists of the rate-tuples for which the rate of any one user cannot be increased without decreasing the rate of at least one other user [4].

At each transmitter, the interference to undesired receivers can be eliminated completely by ZF CB (ZFCB). Due to its simplicity and fully distributed nature, there has been extensive research on ZFCB, e.g., [20]-[22]. The best ZF beamforming vector at transmitter $i$ can be obtained by solving the following optimization problem:

$$
\begin{aligned}
\mathbf{v}_{i}^{*}=\underset{\mathbf{v}_{i} \in \mathbb{C}^{N}}{\arg \max } & \log \left(1+\frac{\left|\mathbf{h}_{i i}^{H} \mathbf{v}_{i}\right|^{2}}{\sigma_{i}^{2}}\right) \\
\text { subject to } & \left|\mathbf{h}_{j i}^{H} \mathbf{v}_{i}\right|=0, \forall j \neq i \text { and }\left\|\mathbf{v}_{i}\right\|^{2} \leq P_{i} .
\end{aligned}
$$

Here, $\left|\mathbf{h}_{j i}^{H} \mathbf{v}_{i}\right|=0$ is the ZF leakage constraint at transmitter $i$ for receiver $j$. If $N \geq K$, the problem (4) has a non-trivial solution and the solution is given by $\mathbf{v}_{i}^{Z F}=c \boldsymbol{\Pi}_{\left[\mathbf{h}_{1 i}, \ldots, \mathbf{h}_{i-1, i}, \mathbf{h}_{i+1, i}, \ldots, \mathbf{h}_{K i}\right]}^{\perp} \mathbf{h}_{i i}$ for some scalar $c$ satisfying the transmit power constraint. In this paper, however, we do not assume that $N \geq K$ necessarily as in the ZF beamforming, but assume that

(A.1) In the case of $N \geq K,\left\{\mathbf{h}_{j i}, j=1, \ldots, K\right\}$ are linearly independent for each $i$. In the case of $N<K$, the element vectors of any subset of $\left\{\mathbf{h}_{j i}, j=1, \ldots, K\right\}$ with cardinality $N$ are linearly independent for each $i$.

Assumption (A.1) is almost surely satisfied for randomly realized channel vectors.

\section{RZF COORDINATED BEAMFORMING IN MISO INTERFERENCE CHANNELS}

\section{A. Formulation}

Although the ZFCB provides an effective way to handling inter-cell interference, the ZFCB is not optimal from the perspective of Pareto optimality, i.e., the rate tuples achieved by ZFCB are in the interior of the achievable rate region [23], and requires the condition $N \geq K$. As mentioned before, even with such complete interference nulling, there exists thermal noise at each receiver, and thus, a certain level of interference leakage comparable to the power of thermal noise can be allowed for better performance. In the MISO IC case, the RZF leakage constraint at transmitter $i$ for receiver $j$ is formulated as follows:

$$
\left|\mathbf{h}_{j i}^{H} \mathbf{v}_{i}\right|^{2} \leq \alpha_{j i} \sigma_{j}^{2}, \quad \forall i, j \neq i,
$$

where $\alpha_{j i} \geq 0$ is a constant ${ }^{3}$ that controls the allowed level of interference leakage from transmitter $i$ to receiver $j$ relative to the thermal noise level $\sigma_{j}^{2}$ at receiver $j$. When $\alpha_{j i}=0$ for all $j \neq i$, the RZF constraints reduce to the conventional ZF constraints. When $\alpha_{j i}>0$, on the other hand, the ZF constraints are relaxed to yield a larger feasible set for $\mathbf{v}_{i}$ than that associated with the ZF constraints and due to this relaxation the condition $N \geq K$ is not necessary anymore.

Under the RZF framework, the power of interference from undesired transmitters at receiver $i$ is upper bounded as

$$
\sum_{j=1, j \neq i}^{K}\left|\mathbf{h}_{i j}^{H} \mathbf{v}_{j}\right|^{2} \leq \sum_{j \neq i} \alpha_{i j} \sigma_{i}^{2}=: \epsilon_{i} \sigma_{i}^{2}
$$

Therefore, a lower bound on the rate of user $i$ under RZF is obtained by using (6) as

$$
\log \left(1+\frac{\left|\mathbf{h}_{i i}^{H} \mathbf{v}_{i}\right|^{2}}{\sigma_{i}^{2}+\sum_{j \neq i}\left|\mathbf{h}_{i j}^{H} \mathbf{v}_{j}\right|^{2}}\right) \geq \log \left(1+\frac{\left|\mathbf{h}_{i i}^{H} \mathbf{v}_{i}\right|^{2}}{\left(1+\epsilon_{i}\right) \sigma_{i}^{2}}\right) .
$$

The lower bound on the rate at each receiver does not depend on the beamforming vectors of undesired transmitters and thus, exploiting the RZF constraints, we can convert the intertwined coordinated beam design problem into a set of separate problems for different users based on the lower bound [17]. The separate problem for each transmitter based on RZF is given as follows [17], [18]:

Problem 1: For each transmitter $i \in\{1, \ldots, K\}$,

$$
\begin{array}{cl}
\underset{\mathbf{v}_{i}}{\operatorname{maximize}} & \log \left(1+\frac{\left|\mathbf{h}_{i i}^{H} \mathbf{v}_{i}\right|^{2}}{\left(1+\epsilon_{i}\right) \sigma_{i}^{2}}\right) \\
\text { subject to } & \left|\mathbf{h}_{j i}^{H} \mathbf{v}_{i}\right|^{2} \leq \alpha_{j i} \sigma_{j}^{2}, \quad \forall j \neq i, \\
& \left\|\mathbf{v}_{i}\right\|^{2} \leq P_{i} .
\end{array}
$$

Then, due to the monotonicity of the logarithm, Problem 1 is equivalent to the following problem:

Problem 2 (The MISO RZFCB Problem): For each transmitter $i \in\{1, \ldots, K\}$,

$$
\begin{array}{cl}
\underset{\mathbf{v}_{i}}{\operatorname{maximize}} & \left|\mathbf{h}_{i i}^{H} \mathbf{v}_{i}\right|^{2} \\
\text { subject to } & \left|\mathbf{h}_{j i}^{H} \mathbf{v}_{i}\right|^{2} \leq \alpha_{j i} \sigma_{j}^{2}, \quad \forall j \neq i, \\
& \left\|\mathbf{v}_{i}\right\|^{2} \leq P_{i} .
\end{array}
$$

From now on, we will consider Problem 2 (the RZFCB problem) and refer to the solution to Problem 2 as the RZF beamforming vector.

\section{B. The Optimality and Solution Structure of RZFCB in MISO Interference Channels}

In this subsection, we will investigate the optimality and structure of the solution to Problem 2. We start with the opti-

\footnotetext{
${ }^{3}$ In the RZF scheme, $\left\{\alpha_{j i}, j, i=1, \ldots, K, j \neq i\right\}$ are system design parameters that should be designed properly for optimal performance. The practical significance of the parameterization in terms of the interference leakage levels will be clear in Section IV-B.
} 
mality of the RZFCB scheme. Without inter-cell interference, it is optimal for the transmitter to use the MF beam vector with full transmit power. However, with inter-cell interference, such a selfish strategy leads to poor performance due to large mutual interference [23]. Thus, to enhance the overall rate performance in the network, the beamforming vector should be designed to be as close as possible to the MF beam vector without giving too much interference to undesired receivers, and this strategy is the RZFCB in Problem 2 (or Problem 1 equivalently). The optimality of the RZFCB is given in the following theorem of Shang et al. [17] or Zhang and Cui [18].

Theorem 1: [18] Any rate-tuple $\left(R_{1}, \ldots, R_{K}\right)$ on the Pareto boundary of the achievable rate region defined in (3) can be achieved by the RZFCB if the levels $\left\{\alpha_{i j} \sigma_{i}^{2}, \forall i, j \neq i\right\}$ of interference leakage are properly chosen.

Proof: See Proposition 3.2 in [18].

Surprisingly, the separate beam design based on the rate lower bound in Problem 2 can achieve any Pareto-optimal point of the achievable rate region if the interference relaxation parameters are well chosen. ${ }^{4}$ It was also shown that Problem 2 and the approach in [5] are two different approaches to the same multi-objective optimization problem [24]. (Furthermore, it is shown that the Pareto boundary of the achievable rate region with partial CSI can also be parametrized by the interference relaxation parameters in the ergodic rate case [25] and in the outage-rate case [26].) Due to Theorem 1, in the MISO IC case, the remaining problems for the RZFCB are i) to construct an efficient algorithm to solve the RZFCB problem for given $\left\{\alpha_{i j} \sigma_{i}^{2}, \forall i, j \neq i\right\}$ and ii) to devise a method to design $\left\{\alpha_{i j} \sigma_{i}^{2}, \forall i, j \neq i\right\}$ for controlling the location of the rate-tuple along the Pareto boundary of the achievable rate region. We will consider Problem 2 for given $\left\{\alpha_{i j} \sigma_{i}^{2}, \forall i, j \neq i\right\}$ here and will consider the rate control problem in the next section.

First, we will derive an efficient algorithm for obtaining a good approximate solution to Problem 2 for given $\left\{\alpha_{i j} \sigma_{i}^{2}, \forall i, j \neq i\right\}$. To do this, we need to investigate the solution structure of the RZFCB problem. Instead of solving Problem 1 as in [18] (this becomes complicated due to the logarithm), we here solve Problem 2, which is equivalent to Problem 1. Note that Problem 2 is not a convex optimization problem since it maximizes a convex cost function under convex constraint sets instead of minimizing the cost. However, Problem 2 can be made an equivalent convex problem by exploiting the phase ambiguity of the solution to Problem 2 and making $\mathbf{h}_{i i}^{H} \mathbf{v}_{i}$ real and nonnegative without affecting the value of $\left|\mathbf{h}_{i i}^{H} \mathbf{v}_{i}\right|$ as follows [27]:

Problem 3: For each transmitter $i \in\{1, \ldots, K\}$,

$$
\begin{array}{cl}
\underset{\mathbf{v}_{i}}{\operatorname{maximize}} & \mathbf{h}_{i i}^{H} \mathbf{v}_{i} \\
\text { subject to } & \left|\mathbf{h}_{j i}^{H} \mathbf{v}_{i}\right|^{2} \leq \alpha_{j i} \sigma_{j}^{2}, \quad \forall j \neq i, \\
& \left\|\mathbf{v}_{i}\right\|^{2} \leq P_{i}, \\
& \mathbf{h}_{i i}^{H} \mathbf{v}_{i} \geq 0 .
\end{array}
$$

Here, the constraint (17) implies $\operatorname{imag}\left(\mathbf{h}_{i i}^{H} \mathbf{v}_{i}\right)=0$ and due to this constraint, maximizing $\left|\mathbf{h}_{i i}^{H} \mathbf{v}_{i}\right|^{2}$ is equivalent to maximizing $\mathbf{h}_{i i}^{H} \mathbf{v}_{i}$.

\footnotetext{
${ }^{4}$ The beamforming vectors from Problem 2 are necessary to achieve a point on the Pareto boundary but not sufficient. An arbitrary choice of parameters $\left\{\alpha_{i j}\right\}$ does not lead to a point on the Pareto boundary.
}

Lemma 1: Let $\mathbf{v}_{i}^{\text {opt }}$ be a solution of the RZFCB problem (i.e., Problem 2) for transmitter $i$. Then, $\mathbf{v}_{i}^{\text {opt }}$ is represented as follows:

$$
\mathbf{v}_{i}^{\mathrm{opt}}=c_{i i} \mathbf{h}_{i i}+\sum_{j \in \Gamma_{i}} c_{j i} \mathbf{h}_{j i}
$$

for some $\left\{c_{j i} \in \mathbb{C}: j \in \Gamma_{i} \cup\{i\}\right\}$, where $\Gamma_{i}:=\{j:$ $\left.\left|\mathbf{h}_{j i}^{H} \mathbf{v}_{i}^{\mathrm{opt}}\right|^{2}=\alpha_{j i} \sigma_{j}^{2}\right\},\left\|\mathbf{v}_{i}^{\mathrm{opt}}\right\|^{2}=P_{i}$ for $N \geq K$, and $\left\|\mathbf{v}_{i}^{\text {opt }}\right\|^{2} \leq P_{i}$ for $N<K$.

Proof: Proof is based on the equivalent formulation in Problem 3. Since Problem 3 is a convex optimization problem, the optimal solution can be obtained by the Karush-Kuhn-Tucker (KKT) conditions. The Lagrangian of Problem 3 for transmitter $i$ is given by

$$
\begin{aligned}
\mathcal{L}\left(\mathbf{v}_{i}, \boldsymbol{\lambda}, \mu, \nu\right)= & -\mathbf{h}_{i i}^{H} \mathbf{v}_{i} \\
& +\sum_{j=1, j \neq i}^{K} \lambda_{j}\left(\left|\mathbf{h}_{j i}^{H} \mathbf{v}_{i}\right|^{2}-\alpha_{j i} \sigma_{j}^{2}\right) \\
& +\mu\left(\left\|\mathbf{v}_{i}\right\|^{2}-P_{i}\right)-\nu \mathbf{h}_{i i}^{H} \mathbf{v}_{i},
\end{aligned}
$$

where $\boldsymbol{\lambda}:=\left\{\lambda_{j} \geq 0: j=1, \ldots, i-1, i+1, \ldots, K\right\}$ and $\mu, \nu \geq 0$ are real dual variables. With optimal dual variables $\boldsymbol{\lambda}^{\star}$, $\mu^{\star}$, and $\nu^{\star}$, the (complex) gradient of the Lagrangian should be zero at $\mathbf{v}_{i}^{\text {opt }}$, i.e.,

$$
\begin{aligned}
\mathbf{0} & =\left.\nabla_{\mathbf{v}_{i}^{\star}} \mathcal{L}\left(\mathbf{v}_{i}, \lambda^{\star}, \mu^{\star}, \nu^{\star}\right)\right|_{\mathbf{v}_{i}=\mathbf{v}_{i}^{\mathrm{opt}}} \\
& =-\mathbf{h}_{i i}+\sum_{j=1, j \neq i}^{K} \lambda_{j}^{\star} \mathbf{h}_{j i} \mathbf{h}_{j i}^{H} \mathbf{v}_{i}^{\mathrm{opt}}+\mu^{\star} \mathbf{v}_{i}^{\mathrm{opt}}-\nu^{\star} \mathbf{h}_{i i} \\
& =-\mathbf{h}_{i i}+\sum_{j \in \Gamma_{i}} \lambda_{j}^{\star} \mathbf{h}_{j i} \mathbf{h}_{j i}^{H} \mathbf{v}_{i}^{\mathrm{opt}}+\mu^{\star} \mathbf{v}_{i}^{\mathrm{opt}}-\nu^{\star} \mathbf{h}_{i i},
\end{aligned}
$$

where $\Gamma_{i}:=\left\{j: \lambda_{j}^{\star}>0\right\}$ and $\nabla_{\mathbf{v}_{i}^{*}}$ is the conjugate Wirtinger gradient. From the complementary slackness condition, $\lambda_{j}^{\star}>0$ only when $\left|\mathbf{h}_{j i}^{H} \mathbf{v}_{i}\right|^{2}=\alpha_{j i} \sigma_{i}^{2}$. Also, from the complementary slackness, we have $\nu^{\star}=0$. Otherwise, $\mathbf{h}_{i i}^{H} \mathbf{v}_{i}^{\text {opt }}=0$ and thus no rate is provided to user $i$. Thus, the gradient of the Lagrangian becomes zero if and only if

$$
\mathbf{h}_{i i}=\left(\mu^{\star} \mathbf{I}+\sum_{j \in \Gamma_{i}} \lambda_{j}^{\star} \mathbf{h}_{j i} \mathbf{h}_{j i}^{H}\right) \mathbf{v}_{i}^{\mathrm{opt}} .
$$

If $\mathbf{Q}:=\left(\mu^{\star} \mathbf{I}+\sum_{j \in \Gamma_{i}} \lambda_{j}^{\star} \mathbf{h}_{j i} \mathbf{h}_{j i}^{H}\right)$ is singular, then $\mathbf{v}_{i}^{\text {opt }}$ exists if and only if $\mathbf{h}_{i i} \in \mathcal{C}(\mathbf{Q})$. However, the condition $\mathbf{h}_{i i} \in \mathcal{C}(\mathbf{Q})$ does not occur almost surely for randomly realized channel vectors, which is assumed here. Therefore, $\mathbf{Q}$ should have full rank for the existence of $\mathbf{v}_{i}^{\text {opt }}$ and the corresponding $\mathbf{v}_{i}^{\text {opt }}$ has two different forms according to the optimal dual variable $\mu^{\star}$.

i) $\mu^{\star}>0$ : This corresponds to the case in which the transmitter uses full power, i.e., $\left\|\mathbf{v}_{i}^{\text {opt }}\right\|^{2}=P_{i}$. In this case, the optimal solution is given by

$$
\mathbf{v}_{i}^{\text {opt }}=\left(\mu^{\star} \mathbf{I}+\sum_{j \in \Gamma_{i}} \lambda_{j}^{\star} \mathbf{h}_{j i} \mathbf{h}_{j i}^{H}\right)^{-1} \mathbf{h}_{i i} .
$$

By applying the matrix inversion lemma recursively, it can be shown that $\mathbf{v}_{i}^{\text {opt }}$ is a linear combination of $\left\{\mathbf{h}_{j i}: j \in \Gamma_{i}^{\prime}:=\right.$ $\left.\Gamma_{i} \cup\{i\}\right\}$. Thus, the solution is represented as (18).

ii) $\mu^{\star}=0$ : This case corresponds to the case in which full power is not used at transmitter $i$. In this case, 
$\mathbf{Q}=\sum_{j \in \Gamma_{i}} \lambda_{j}^{\star} \mathbf{h}_{j i} \mathbf{h}_{j i}^{H}$. The matrix $\mathbf{Q}$ in this case is non-singular if and only if $\left|\Gamma_{i}\right| \geq N$ (i.e., $K>N$ ) under the assumption (A.1), and the corresponding solution is given by

$$
\mathbf{v}_{i}^{\mathrm{opt}}=\left(\sum_{j \in \Gamma_{i}} \lambda_{j}^{\star} \mathbf{h}_{j i} \mathbf{h}_{j i}^{H}\right)^{-1} \mathbf{h}_{i i} .
$$

In this case, $\left\{\mathbf{h}_{i j}, j \in \Gamma_{i}\right\}$ alone span $\mathbb{C}^{N}$ fully and it is therefore clear that the solution is represented as (18). Indeed, any subset of $\left\{\mathbf{h}_{j i}, j=1, \ldots, K\right\}$ with cardinality $N$ forms a full basis for $\mathbb{C}^{N}$ under the assumption (A.1) in this case.

Furthermore, when $N \geq K, \mathbf{v}_{i}^{Z F}$ is feasible and thus, we can always increase power and rate without causing interference to the undesired receivers. Therefore, the optimal solution uses full power, i.e., $\left\|\mathbf{v}_{i}^{\mathrm{opt}}\right\|^{2}=P_{i}$ when $N \geq K$. On the other hand, when $N<K$, we can have either $\mu^{\star}>0\left(\left\|\mathbf{v}_{i}^{\mathrm{opt}}\right\|^{2}=P_{i}\right)$ or $\mu^{\star}=0\left(\left\|\mathbf{v}_{i}^{\text {opt }}\right\|^{2}<P_{i}\right)$.

The solution to RZFCB for a given set of interference relaxation levels is a linear combination of the desired channel and a subset of interference channels for which the RZF constraint (12) is satisfied with equality. Furthermore, it was shown that the interference leakage levels should be designed to make the RZF interference leakage constraints be satisfied tightly in order to achieve a point on the Pareto boundary [18]. In this case, $\Gamma_{i}=\{1, \ldots, K\} \backslash\{i\}$ and thus, the RZF beam structure in Lemma 1 coincides with the Pareto-optimal beam structure derived by Jorswieck et al. in [4]. Now, based on Lemma 1, we present a new useful representation of $\mathbf{v}_{i}^{\text {opt }}$ that provides a clear insight into the RZFCB solution and a basis for fast algorithm construction.

Theorem 2: For transmitter $i$, the RZFCB solution can also be expressed as

$$
\begin{aligned}
\mathbf{v}_{i}^{\text {opt }}=c_{0} \frac{\mathbf{h}_{i i}}{\left\|\mathbf{h}_{i i}\right\|}+c_{1} \frac{\Pi_{\mathbf{A}_{1}}^{\perp} \mathbf{h}_{i i}}{\left\|\boldsymbol{\Pi}_{\mathbf{A}_{1}}^{\perp} \mathbf{h}_{i i}\right\|} & +\cdots \\
& +c_{\left|\tilde{\Gamma}_{i}\right|} \frac{\boldsymbol{\Pi}_{\mathbf{A}_{\left|\tilde{\Gamma}_{i}\right|}}^{\perp} \mathbf{h}_{i i}}{\left\|\Pi_{\mathbf{A}_{\left|\tilde{\Gamma}_{i}\right|}}^{\perp} \mathbf{h}_{i i}\right\|},
\end{aligned}
$$

where $c_{j} \in \mathbb{C}, j=0,1, \ldots,\left|\tilde{\Gamma}_{i}\right|$ and $\mathbf{A}_{j}$ is constructed recursively as

$$
\mathbf{A}_{j}:=\left[\mathbf{A}_{j-1}, \mathbf{h}_{\tilde{\Gamma}_{i}(j), i}\right], \quad j=1, \ldots,\left|\tilde{\Gamma}_{i}\right| .
$$

Here for convenience we let $\mathbf{A}_{0}$ be an $N \times 0$ 'matrix'. $\tilde{\Gamma}_{i}$ is a set made by permuting the elements of $\Gamma_{i}$ according to an arbitrary order, and $\tilde{\Gamma}_{i}(j)$ denotes the $j$-th element of $\tilde{\Gamma}_{i}$.

Proof: From Lemma 1, we know that $\mathbf{v}_{i}^{\text {opt }} \in$ $\mathcal{C}\left(\left[\mathbf{h}_{j i}\right]_{j \in \Gamma_{i}^{\prime}}\right)$. Proof of the theorem is given by showing the equivalence of the two subspaces $\mathcal{C}\left(\left[\mathbf{h}_{j i}\right]_{j \in \Gamma_{i}^{\prime}}\right)$ and $\mathcal{C}\left(\left[\mathbf{h}_{i i}, \boldsymbol{\Pi}_{\mathbf{A}_{1}}^{\perp} \mathbf{h}_{i i}, \ldots, \boldsymbol{\Pi}_{\mathbf{A}_{\left|\tilde{r}_{i}\right|}}^{\perp} \mathbf{h}_{i i}\right]\right)$.

Case (i). $\left|\Gamma_{i}\right|\left(=\left|\tilde{\Gamma}_{i}\right|\right) \leq N-1$ : In this case, $\left\{\mathbf{h}_{i i}, \boldsymbol{\Pi}_{\mathbf{A}_{1}}^{\perp} \mathbf{h}_{i i}, \ldots, \boldsymbol{\Pi}_{\mathbf{A}_{\left|\tilde{\Gamma}_{i}\right|}}^{\perp} \mathbf{h}_{i i}\right\}$ are linearly independent. This is easily shown by replacing $\boldsymbol{\Pi}_{\mathbf{A}_{j}}^{\perp}$ with $\mathbf{I}-\boldsymbol{\Pi}_{\mathbf{A}_{j}}$ and by using the linear independence of $\left\{\mathbf{h}_{j i}\right\}_{j \in \Gamma_{i}^{\prime}}$. Thus, the dimension of $\mathcal{C}\left(\left[\mathbf{h}_{i i}, \boldsymbol{\Pi}_{\mathbf{A}_{1}}^{\perp} \mathbf{h}_{i i}, \ldots, \boldsymbol{\Pi}_{\hat{\mathbf{A}}_{\left|\mathbf{\Gamma}_{j}\right|}}^{\perp} \mathbf{h}_{i i}\right]\right)$ is $\left(\left|\tilde{\Gamma}_{i}\right|+1\right)$, which is the same as that of $\mathcal{C}\left(\left[\mathbf{h}_{j i}\right]_{j \in \Gamma_{i}^{\prime}}\right)$. The projection of any vector in $\mathcal{C}\left(\left[\mathbf{h}_{i i}, \boldsymbol{\Pi}_{\mathbf{A}_{1}}^{\perp} \mathbf{h}_{i i}, \ldots, \boldsymbol{\Pi}_{\mathbf{A}_{|\tilde{\mathbf{\Gamma}}|} \mid}^{\perp} \mathbf{h}_{i i}\right]\right)$ onto the orthogonal complement of $\mathcal{C}\left(\left[\mathbf{h}_{j i}\right]_{j \in \Gamma_{i}^{\prime}}\right)$ is shown in (26).

$$
\begin{aligned}
& \boldsymbol{\Pi}_{\left[\mathbf{h}_{j i}\right]_{j \in \Gamma^{\prime}}{ }_{i}^{\prime}}^{\perp}\left(c_{0} \mathbf{h}_{i i}+c_{1} \boldsymbol{\Pi}_{\mathbf{A}_{1}}^{\perp} \mathbf{h}_{i i}+\cdots+c_{\left|\tilde{\Gamma}_{i}\right|} \boldsymbol{\Pi}_{\mathbf{A}_{\left|\tilde{\Gamma}_{i}\right|}}^{\perp} \mathbf{h}_{i i}\right) \\
& =\boldsymbol{\Pi}_{\left[\mathbf{h}_{j i}\right]_{j \in \Gamma_{i}^{\prime}}}^{\perp}\left(c_{0} \mathbf{h}_{i i}+c_{1}\left(\mathbf{I}-\mathbf{\Pi}_{\mathbf{A}_{1}}\right) \mathbf{h}_{i i}+\cdots\right. \\
& \left.+c_{\left|\tilde{\Gamma}_{i}\right|}\left(\mathbf{I}-\boldsymbol{\Pi}_{\mathbf{A}_{\left|\tilde{\Gamma}_{i}\right|}}\right) \mathbf{h}_{i i}\right) \\
& =\boldsymbol{\Pi}_{\left[\mathbf{h}_{j i}\right]_{j \in \Gamma_{i}^{\prime}}}^{\perp}\left(c_{0} \mathbf{h}_{i i}+c_{1}\left(\mathbf{I}-\boldsymbol{\Pi}_{\mathbf{h}_{\tilde{\Gamma}_{i}(1), i}}\right) \mathbf{h}_{i i}+\cdots\right. \\
& \left.+c_{\left|\tilde{\Gamma}_{i}\right|}\left(\mathbf{I}-\boldsymbol{\Pi}_{\left[\mathbf{h}_{\tilde{\Gamma}_{i}(j), i}\right]_{j=1}^{\left|\tilde{\Gamma}_{i}\right|}}\right) \mathbf{h}_{i i}\right) \\
& =\Pi_{\left[\mathbf{h}_{j i}\right]_{j \in \Gamma_{i}^{\prime}}}^{\perp}\left(\sum_{j=0}^{\left|\tilde{\Gamma}_{i}\right|} c_{j} \mathbf{h}_{i i}-c_{1} \boldsymbol{\Pi}_{\mathbf{h}_{\tilde{\Gamma}_{i}(1), i}} \mathbf{h}_{i i}-\cdots\right. \\
& \left.-c_{\left|\tilde{\Gamma}_{i}\right|} \boldsymbol{\Pi}_{\left[\mathbf{h}_{\tilde{\Gamma}_{i}(j), i}\right]_{j=1}^{\left|\tilde{\Gamma}_{i j}\right|}} \mathbf{h}_{i i}\right)=0 .
\end{aligned}
$$

By (26) the orthogonal complement of $\mathcal{C}\left(\left[\mathbf{h}_{i i}, \boldsymbol{\Pi}_{\mathbf{A}_{1}}^{\perp} \mathbf{h}_{i i}, \ldots\right.\right.$, $\left.\left.\boldsymbol{\Pi}_{\mathbf{A}_{\left|\tilde{\Gamma}_{i}\right|}}^{\perp} \mathbf{h}_{i i}\right]\right)$ is included in that of $\mathcal{C}\left(\left[\mathbf{h}_{j i}\right]_{j \in \Gamma_{i}^{\prime}}\right)$, but $\mathcal{C}\left(\left[\mathbf{h}_{i i}, \boldsymbol{\Pi}_{\mathbf{A}_{1}}^{\perp} \mathbf{h}_{i i}, \ldots, \boldsymbol{\Pi}_{\mathbf{A}_{\left|\tilde{\Gamma}_{i}\right|}}^{\perp} \mathbf{h}_{i i}\right]\right)$ and $\mathcal{C}\left(\left[\mathbf{h}_{j i}\right]_{j \in \Gamma_{i}^{\prime}}\right)$ have the same dimensions. Thus, the two orthogonal complements are the same, and hence, the two subspaces themselves are the same. Consequently, for any $c_{i i} \mathbf{h}_{i i}+\sum_{j \in \Gamma_{i}} c_{j i} \mathbf{h}_{j i}$ with arbitrary $\left\{c_{j i} \in \mathbb{C}: j \in \Gamma_{i}^{\prime}\right\}$, there exists some $\left\{c_{j} \in \mathbb{C}: 0 \leq j \leq\left|\tilde{\Gamma}_{i}\right|\right\}$ s.t.

$$
\mathbf{v}_{i}^{\text {opt }}=c_{i i} \mathbf{h}_{i i}+\sum_{j \in \Gamma_{i}} c_{j i} \mathbf{h}_{j i}=c_{0} \frac{\mathbf{h}_{i i}}{\left\|\mathbf{h}_{i i}\right\|}+\sum_{j=1}^{\prime \tilde{\Gamma}_{i} \mid} c_{j} \frac{\boldsymbol{\Pi}_{\mathbf{A}_{j}}^{\perp} \mathbf{h}_{i i}}{\left\|\boldsymbol{\Pi}_{\mathbf{A}_{j}}^{\perp} \mathbf{h}_{i i}\right\|} .
$$

Case (ii). $\left|\Gamma_{i}\right| \geq N$ : In this case, both $\left\{\mathbf{h}_{j i}, j \in \Gamma_{i}^{\prime}\right\}$ and $\left\{\mathbf{h}_{i i}, \boldsymbol{\Pi}_{\mathbf{A}_{1}}^{\perp} \mathbf{h}_{i i}, \ldots, \boldsymbol{\Pi}_{\mathbf{A}_{\left|\tilde{\Gamma}_{j}\right|}}^{\perp} \mathbf{h}_{i i}\right\}$ span the whole $\mathbb{C}^{N}$. Thus, the claim is trivially satisfied.

Theorem 2 states that the RZF solution is a linear combination of vectors that are obtained by projecting the desired channel vector onto the orthogonal complements of a series of subspaces spanned by the channels from the transmitter to the undesired receivers. Furthermore, the series of subspaces are obtained by sequentially including one additional interference channel vector at a time, as shown in (25). Soon, it will be shown that, to obtain the RZF solution to Problem 2, the order of interference channel inclusion for constructing $\mathbf{A}_{j}$ s in Theorem 2 is determined by the set of allowed interference levels and the channel realization.

\section{The Sequential Orthogonal Projection Combining Method and Closed-Form Solutions}

In this subsection, we propose an efficient beam design method for RZFCB that successively allocates the transmit power to certain vectors obtained by sequential orthogonal projection of the desired channel vector onto monotonically decreasing subspaces. Furthermore, we provide the closed-form solution to the RZFCB problem in the two-user case and an approximate closed-form solution in the three-user case.

To obtain the RZF beamforming vector under given interference relaxation constraints for a given channel realization, Problem 2 should be solved. One can use a numerical method [28], as in [18]. However, such a method requires a numerical search for determining the Lagrange dual variables satisfying 
the RZF constraints and the transmit power constraint. To circumvent such difficulty and to increase the practicality of the RZFCB, we exploit Theorem 2 to construct an efficient method to find the RZFCB solution. Theorem 2 provides us with a very convenient way of obtaining the RZFCB solution for given interference leakage levels for a given channel realization; we only need to find $\tilde{\Gamma}_{i}$ and complex coefficients $\left\{c_{i}\right\}$ in (24) for each transmitter. The idea is based on the fact that the RZF beamforming vector should be designed to be as close as possible to the MF beam vector under the interference leakage constraints for the maximum rate under RZF, as described in Problem 2. Hereafter, we will explain how the coefficients $\left\{c_{i}\right\}$ and the matrices $\left\{\mathbf{A}_{i}\right\}$ in Theorem 2 can be obtained to maximize the rate under the RZF interference and power constraints. Consider transmitter $i$ without loss of generality. For the given transmit power constraint $\left\|\mathbf{v}_{i}\right\|^{2} \leq P_{i}$, it may not be possible to allocate all of the transmit power to the MF direction $\mathbf{h}_{i i}$ because this allocation may violate the RZF constraints. The rate greedy approach under the RZF constraints for a given channel realization is explained as follows. First, we should start to allocate the transmit power to the direction of $\mathbf{h}_{i i}$ by increasing $c_{0}$ with some phase until this allocation hits one of the RZF constraints with equality, i.e., the interference level to one of the undesired receivers reaches the allowed maximum exactly. (In the case that the allowed interference levels to all undesired receivers are the same, this receiver is the receiver whose channel vector has the maximum inner product with $\mathbf{h}_{i i}$.) The index of this receiver is $\tilde{\Gamma}_{i}(1)$. At this point, transmitter $i$ cannot allocate the transmit power to the direction $\mathbf{h}_{i i}$ anymore since this would violate the RZF constraint for receiver $\tilde{\Gamma}_{i}(1)$. Since the RZF constraints for other undesired receivers are still met with strict inequality, transmitter $i$ can still cause interference to the remaining receivers. Thus, for the maximum rate under the RZF constraints, transmitter $i$ should now start to allocate the remaining power to the direction of $\boldsymbol{\Pi}_{\mathbf{A}_{1}}^{\perp} \mathbf{h}_{i i}$, where $\mathbf{A}_{1}=\left[\mathbf{h}_{\tilde{\Gamma}_{i}(1), i}\right]$, until this allocation hits another RZF constraint with equality. The index of this receiver is $\tilde{\Gamma}_{i}(2)$. (Note that $\boldsymbol{\Pi}_{\mathbf{A}_{1}}^{\perp} \mathbf{h}_{i i}$ is the direction of maximizing the data rate without causing additional interference to receiver $\tilde{\Gamma}_{i}(1)$.) Now, transmitter $i$ cannot cause interference to receiver $\tilde{\Gamma}_{i}(2)$ in addition to receiver $\tilde{\Gamma}_{i}(1)$ anymore. Therefore, at this point, transmitter $i$ should start to allocate its remaining power to the next greedy direction $\boldsymbol{\Pi}_{\mathbf{A}_{2}}^{\perp} \mathbf{h}_{i i}$, where $\mathbf{A}_{2}=\left[\mathbf{h}_{\tilde{\Gamma}_{i}(1), i}, \mathbf{h}_{\tilde{\Gamma}_{i}(2), i}\right]$. This greedy power allocation without violating the RZF constraints should be done until either all the transmit power is used up $\left(\mu^{\star}>0\right.$ in Lemma 1) or we cannot find a new direction that does not cause interference to the users that are already in the set $\tilde{\Gamma}_{i}$ ( $\mu^{\star}=0$ in Lemma 1 ). When $N \geq K$ and transmit power still remains even after hitting all the $K-1$ interference leakage constraints with equality, from then on, all the remaining power should be allocated to the ZF direction. This coincides with our intuition that $\mathrm{ZF}$ is optimal at a high signal-to-noise ratio (SNR) in the case of $N>K$. On the other hand, when all the transmit power is used up before reaching the remaining interference constraints with equality, the corresponding remaining interference channel vectors do not appear in the solution. The final RZF solution is the sum of these component vectors and has the form in (24). In this way, the RZFCB solution can be obtained by combining the sequential projections of the desired channel vector $\mathbf{h}_{i i}$ onto the orthogonal complements of the subspaces $\mathcal{C}\left(\mathbf{A}_{1}\right) \subset \cdots \subset \mathcal{C}\left(\mathbf{A}_{\left|\tilde{\Gamma}_{i}\right|}\right)$. Thus, we refer to this beam design method as the sequential orthogonal projection combining (SOPC) method. 5 By Theorem 1, the SOPC strategy with a well chosen set of interference relaxation levels is Pareto-optimal for MISO K-pair interference channels with single-user decoding.

An interesting interpretation of the SOPC strategy is in an analogy with the water-filling strategy. The water-filling strategy distributes power to resource bins according to the effectiveness of each bin, and the power fills into the bin with the lowest noise level (or the most effective bin) first. Similarly, the SOPC strategy allocates power to the most effective direction first and then the next most effective direction when the first direction cannot accommodate power anymore. This procedure continues until either the procedure uses up the power or it cannot find a new feasible direction. So, the SOPC strategy can be viewed graphically as pouring water on top of a multi-tiered fountain, as illustrated in Fig. 1. The relationship of the RZFCB/SOPC design and the two-user result by Jorswieck et al. [4] is explained in Fig. 2. In the two user case, Jorswieck et al. have shown that a Pareto-optimal beam vector is a convex combination of the MF beam $\mathbf{v}_{i}^{M F}$ and the ZF beam $\mathbf{v}_{i}^{Z F}$ satisfying the power constraint, i.e., $\mathbf{v}_{i}=\sqrt{P_{i}} \frac{\lambda_{i} \mathbf{v}_{i}^{M F}+\left(1-\lambda_{i}\right) \mathbf{v}_{i}^{Z F}}{\left\|\lambda_{i} \mathbf{v}_{i}^{M F}+\left(1-\lambda_{i}\right) \mathbf{v}_{i}^{Z F}\right\|}$, where $0 \leq \lambda_{i} \leq 1$. Thus, the feasible set of optimal beam vectors is the arc denoted by $\mathcal{F}$ in Fig. 2. All the points on this arc can be represented by the sum of the two vectors in red, and the size of the component vector in the MF direction is determined by its projection onto $\mathcal{C}\left(\mathbf{h}_{21}\right)$, i.e., the allowed interference level to the other receiver in the RZF context. Thus, the two-user result by Jorswieck et al. can be viewed as a special case of the SOPC strategy when the number of users is two. The key difference is the parameterization; $\alpha_{12}$ and $\alpha_{21}$ are the parameters in the RZF framework whereas the linear combining coefficients $\lambda_{1}$ and $\lambda_{2}$ are the parameters in [4].

Now consider the detailed implementation of the SOPC method. Before considering the general case of an arbitrary number $K$ of users, we consider simple two-user and three-user cases. Here, we restrict the combining coefficients $\left\{c_{i}\right\}$ to the set of real numbers. It will shortly be shown that the performance loss caused by restricting $\left\{c_{i}\right\}$ to real numbers is negligible. Furthermore, it is the optimal solution of the RZFCB when $K=2$. For simplicity, we only provide the solution for transmitter 1 . The solutions for other transmitters can be obtained in a similar way.

Proposition 1: The closed-form SOPC solution in the twopair MISO IC case is given by

$$
\mathbf{v}_{1}= \begin{cases}\sqrt{P_{1}} \mathbf{v}_{1}^{M F}, & \text { if } P_{1} \leq \frac{\alpha_{21} \sigma_{2}^{2}}{\left|\mathbf{h}_{21}^{H} \mathbf{v}_{1}^{M F}\right|^{2}}, \\ \xi_{0} \mathbf{v}_{1}^{M F}+\xi_{1} \mathbf{v}_{1}^{Z F}, & \text { otherwise, }\end{cases}
$$

${ }^{5}$ The rate optimality of the SOPC strategy under the RZF constraints is straightforward to see. Suppose that we are given any beam vector that is a linear combination of $\left\{\mathbf{h}_{j i}\right\}$, satisfies the RZF interference and power constraints but is not the SOPC solution. Then, the vector can still be represented in terms of the SOPC basis in Theorem 2 and some of the basis component vectors with larger inner product with the MF direction do not satisfy the RZF constraints with equality. Thus, the rate can be increased by allocating power from the basis component vector with smaller inner product with the MF direction to the basis component vector with larger inner product with the MF direction until the RZF constraints are satisfied with equality. 


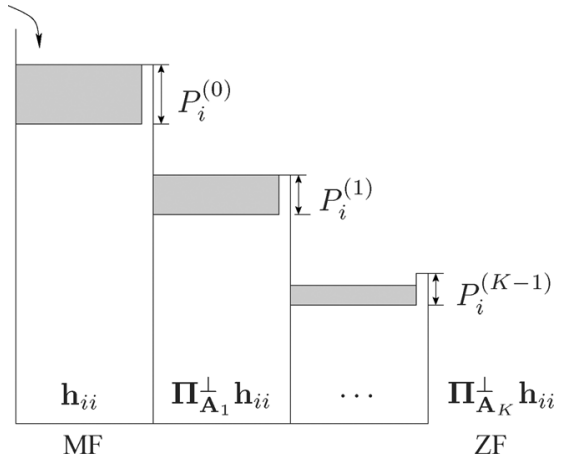

Fig. 1. The SOPC strategy in the case of $N \geq K$ : Water-pouring on a multitiered fountain.

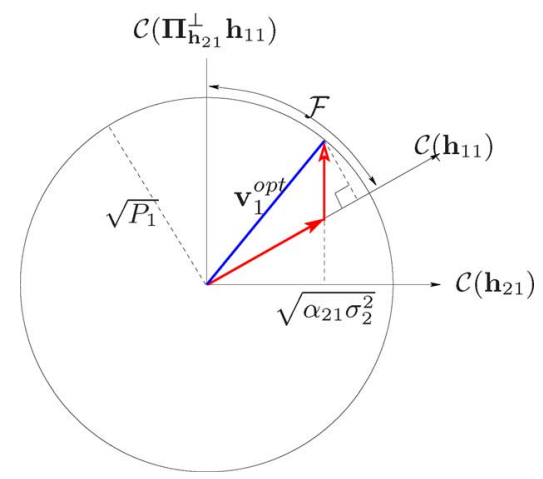

Fig. 2. SOPC interpretation of the two-user result.

where $\mathbf{v}_{1}^{M F}=\frac{\mathbf{h}_{11}}{\left\|\mathbf{h}_{11}\right\|}, \mathbf{v}_{1}^{Z F}=\frac{\boldsymbol{\Pi}_{\mathbf{h}_{21}}^{\perp} \mathbf{h}_{11}}{\left\|\mathbf{\Pi}_{\mathbf{h}_{21}}^{\perp} \mathbf{h}_{11}\right\|}, \xi_{0}=\sqrt{\frac{\alpha_{21} \sigma_{2}^{2}}{\mid \mathbf{h}_{21}^{H} \mathbf{v}_{1}^{M F^{\prime} \mid 2}}}$, and $\xi_{1}=-\rho \xi_{0}+\sqrt{P_{1}-\xi_{0}^{2}\left(1-\rho^{2}\right)}$. Here, $\rho=$ $\left(\mathbf{v}_{1}^{M F}\right)^{H} \mathbf{v}_{1}^{Z F}=\left\|\boldsymbol{\Pi}_{\mathbf{h}_{21}}^{\perp} \mathbf{h}_{11}\right\| /\left\|\mathbf{h}_{11}\right\| \in \mathbb{R}_{+}$.

Proof: Proof of Proposition 1 can be found in [14].

In the case of $K \geq 3$, it is cumbersome to distinguish all possible scenarios for deriving an explicit SOPC solution. Thus, we propose an algorithm implementing the SOPC strategy with real combining coefficients in Table I. In the general case of $K \geq 3$, the implementation of the SOPC algorithm can be simplified by the known result in the Kalman filtering theory, provided in the following lemma.

Lemma 2 (Sequential Orthogonal Projection [29]): Let $\mathcal{H}$ be a Hilbert space with norm $\|\cdot\|$ and inner product $\langle\cdot, \cdot\rangle$. Consider $\mathbf{x} \in \mathcal{H}$ and a closed linear subspace $\mathbf{A}_{j}$ of $\mathcal{H}$. For some $\mathbf{y} \in \mathcal{H}$ but $\mathbf{y} \notin \mathbf{A}_{j}$, the following equality holds

$$
\begin{aligned}
\boldsymbol{\Pi}_{\mathbf{A}_{j+1}} \mathbf{x} & =\boldsymbol{\Pi}_{\left[\mathbf{A}_{j}, \mathbf{y}\right]} \mathbf{x} \\
& =\boldsymbol{\Pi}_{\mathbf{A}_{j}} \mathbf{x}+\frac{\left\langle\mathbf{x}-\boldsymbol{\Pi}_{\mathbf{A}_{j}} \mathbf{x}, \mathbf{y}-\boldsymbol{\Pi}_{\mathbf{A}_{j}} \mathbf{y}\right\rangle}{\left\|\mathbf{y}-\boldsymbol{\Pi}_{\mathbf{A}_{j}} \mathbf{y}\right\|^{2}}\left(\mathbf{y}-\boldsymbol{\Pi}_{\mathbf{A}_{j}} \mathbf{y}\right) .
\end{aligned}
$$

Since we need to compute $\boldsymbol{\Pi}_{\mathbf{A}_{j}}^{\perp} \mathbf{h}_{i i}=\left(\mathbf{I}-\boldsymbol{\Pi}_{\mathbf{A}_{j}}\right) \mathbf{h}_{i i}$ in the SOPC algorithm, Lemma 2 can be applied recursively by exploiting the fact $\mathbf{A}_{j}=\left[\mathbf{A}_{j-1}, \mathbf{h}_{\tilde{\Gamma}(j), i}\right]$. Thus, we only need to compute $\boldsymbol{\Pi}_{\mathbf{A}_{j-1}} \mathbf{h}_{\tilde{\Gamma}(j), i}$ for each $j \in\{1,2, \ldots, K\}$. The proposed algorithm in Table I computes the direction and size of the component vector for SOPC directly in each step.
TABLE I

The Sequential Orthogonal Projection Combining Algorithm
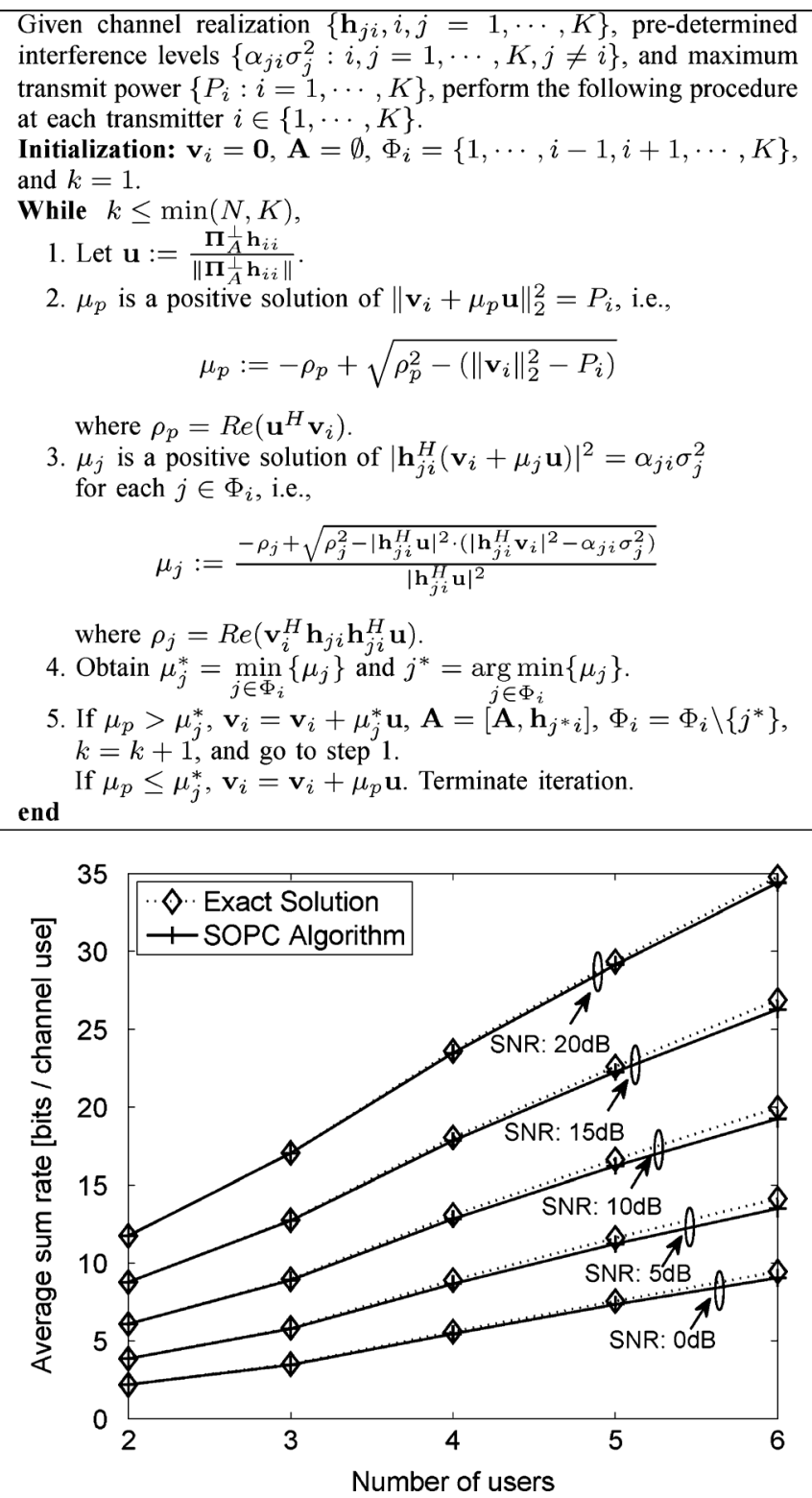

Fig. 3. Average sum rates of the exact RZFCB solution and the proposed SOPC algorithm with real coefficients. (Here, $N=K$ and the average sum rate is obtained over 50 i.i.d. channel realizations).

The proposed SOPC solution based on real coefficients is a sub-optimal solution to the RZFCB problem in the case of $K \geq 3$. However, the performance loss between the optimal RZFCB (or exact SOPC) beamforming vector and the proposed SOPC solution based on real coefficients is insignificant for a wide range of meaningful SNR values, as seen in Fig. 3. Thus, practically, the proposed SOPC solution can be used with negligible performance loss. Note that the necessary computations for the proposed SOPC solution are a few inner product and square root operations and the complexity of the SOPC method is simply $O(N)$, where $N$ is the number of transmit antennas at the transmitter. The proposed SOPC method reduces computational complexity to obtain an RZF solution by order of hundreds when compared to the ellipsoid method for the RZFCB 


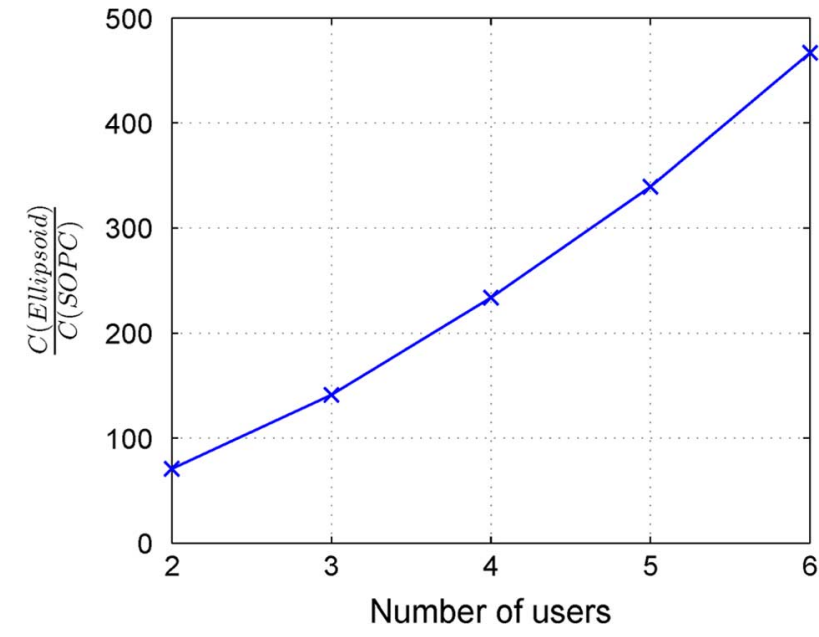

Fig. 4. Computational complexity for RZFCB beam design: Ellipsoid method [18], [28] versus $\operatorname{SOPC}(N=K$ and $\operatorname{SNR}=5 \mathrm{~dB})$.

solution used in [18], as shown in Fig. 4, and the solution procedure can easily be programmed in a real hardware. ${ }^{6}$

\section{RATE-Tuple CONTROL}

In the previous section, we provided an $O(N)$-complexity algorithm to solve the RZFCB problem for a given set $\left\{\alpha_{j i}\right\}$ of interference relaxation parameters. Now, we consider how to design these parameters in order to control the rate-tuple. Although the Pareto-boundary achievaing parameter design problem is fully solved based on the Walrasian equilibrium theory in the case of two users [30], the parameter design problem for Pareto optimality still remains in the general case with more than two users. Here, we provide two approaches suitable for designing the interference relaxation parameters under the RZFCB framework. We first provide a centralized approach to determine $\left\{\alpha_{j i}\right\}$ with the aim of controlling the rate-tuple along the Pareto boundary of the achievable rate region and then a fully-distributed heuristic approach that exploits the parameterization in terms of interference relaxation levels in RZFCB and is able to control the rate-tuple location roughly along the Pareto boundary of the achievable rate region.

\section{A. A Centralized Approach}

By Theorem 1, with a set of well chosen allowed interference leakage levels, the RZFCB can achieve any Pareto-optimal point of the rate region. However, the problem of designing the interference leakage levels $\left\{\alpha_{j i}\right\}$ in the network remains. Under the RZFCB framework, in [18], a necessary condition for the interference relaxation parameters at each receiver to achieve a Pareto-optimal point was derived. Based on the necessary condition, the authors proposed an iterative algorithm that updates the interference relaxation parameters. Although the algorithm in [18] is applicable to general $K$-user MISO interference channels, it cannot control the rate-tuple location on the Pareto boundary to which the algorithm converges. To control the rate-tuple to an arbitrary point along the Pareto-boundary

\footnotetext{
${ }^{6}$ The case of $K=3$ is particularly important when the hexagonal cell structure is used and three cells are coordinating their beam vectors. An approximate closed-form solution can be obtained in this case and is available in [14].
}

TABLE II

A Centralized Algorithm FOR Determining $\left\{\alpha_{j i}\right\}$

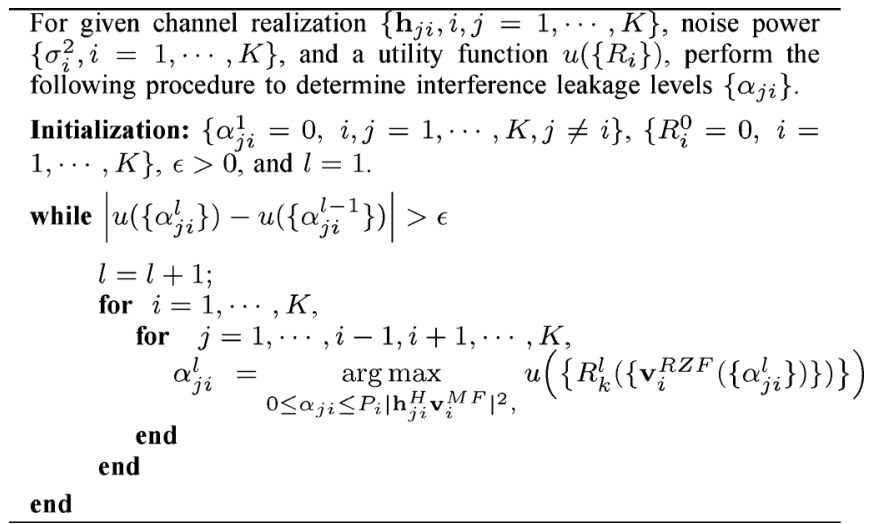

of the achievable rate region, we here apply the utility function based approach in [31] to the RZF parameterization in terms of interference leakage levels.

Exploiting the fact that the RZFCB can achieve any Paretoboundary point by adjusting $\left\{\alpha_{j i}\right\}$, we convert the problem of finding a desired point on the Pareto boundary of the achievable rate region into that of finding an optimal point of the following optimization problem:

$$
\begin{array}{cc}
\underset{\left\{\alpha_{j i}\right\}}{\operatorname{maximize}} & u\left(R_{1}\left(\left\{\mathbf{v}_{i}^{R Z F}\left(\left\{\alpha_{j i}\right\}\right)\right\}\right), \ldots,\right. \\
& \left.R_{K}\left(\left\{\mathbf{v}_{i}^{R Z F}\left(\left\{\alpha_{j i}\right\}\right)\right\}\right)\right), \\
\text { subject to } & \left|\mathbf{h}_{j i}^{H} \mathbf{v}_{i}\right|^{2} \leq \alpha_{j i} \sigma_{j}^{2}, \quad \forall i, j \neq i \\
& \left\|\mathbf{v}_{i}\right\|^{2} \leq P_{i}, \quad \forall i
\end{array}
$$

where $u\left(R_{1}, \ldots, R_{K}\right)$ is the desired utility function and several examples include the weighted sum rate $u\left(R_{1}, \ldots, R_{K}\right)=\sum w_{i} R_{i}$, where $w_{i} \geq 0$ and $\sum w_{i}=1$, the Nash bargaining point $u\left(R_{1}, \ldots, R_{K}\right)=\prod_{i=1}^{K}\left(R_{i}-R_{i}^{N E}\right)$, where $R_{i}^{N E}=\log _{2}\left(1+\frac{\left|\mathbf{h}_{i i}^{H} \mathbf{M}_{i}^{M F}\right|^{2}}{\sigma_{i}^{2}+\sum_{j \neq i}\left|\mathbf{h}_{i j}^{H} \mathbf{v}_{j}^{M F}\right|^{2}}\right)$, and the egalitarian point $u\left(R_{1}, \ldots, R_{K}\right)=\min \left(R_{1}, \ldots, R_{K}\right)$ [31]. The optimization (28) can be solved by an alternating optimization technique. That is, we fix all other $\alpha_{j i}$ 's except one interference relaxation parameter and update the unfixed parameter so that the utility function is maximized. After this update, the next $\alpha_{j i}$ is picked for update. This procedure continues until converges. The proposed algorithm is described in detail in Table II. For a given utility function $u\left(R_{1}, \ldots, R_{K}\right)$, the RZF beam vectors $\left\{\mathbf{v}_{i}^{R Z F}\right\}$ can be obtained as functions of $\left\{\alpha_{j i}\right\}$ by the SOPC method, the rate-tuple can be computed as a function of $\left\{\mathbf{v}_{i}^{R Z F}\right\}$ by (2), and finally the utility function value can be computed as a function of $\left(R_{1}, \ldots, R_{K}\right)$. Thus, the utility value as a function of $\left\{\alpha_{j i}\right\}$ can be computed very efficiently by the SOPC method for the proposed centralized algorithm, and this fact makes it easy to apply a numerical optimization method such as the interior point method to the per-iteration optimization in Table II.

Due to the non-convexity of utility functions w.r.t. $\left\{\alpha_{j i}\right\}$, the convergence of the proposed algorithm to the global optimum is not guaranteed, but the proposed algorithm converges to a locally optimal point by the monotone convergence theorem since 


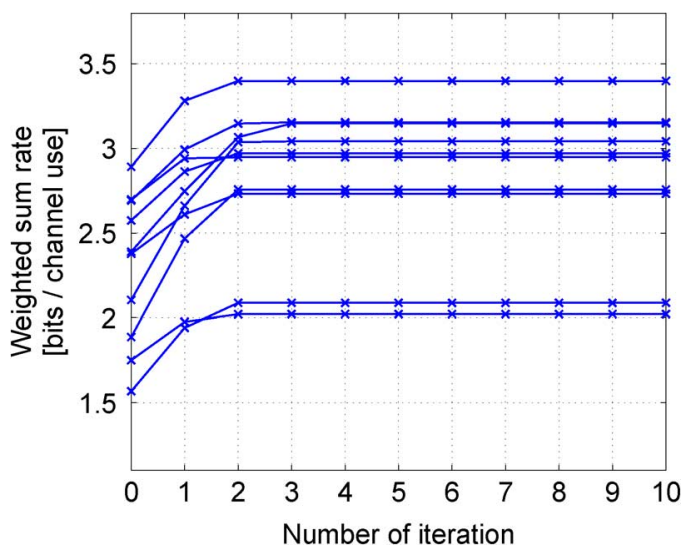

Fig. 5. Convergence of the proposed centralized approach ( $K=N=3$, $P_{i}=10, \sigma_{i}=1$ for all $i=1,2,3$.)

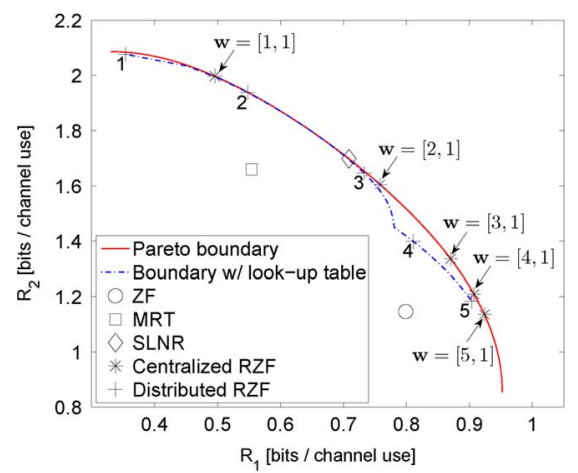

(a)

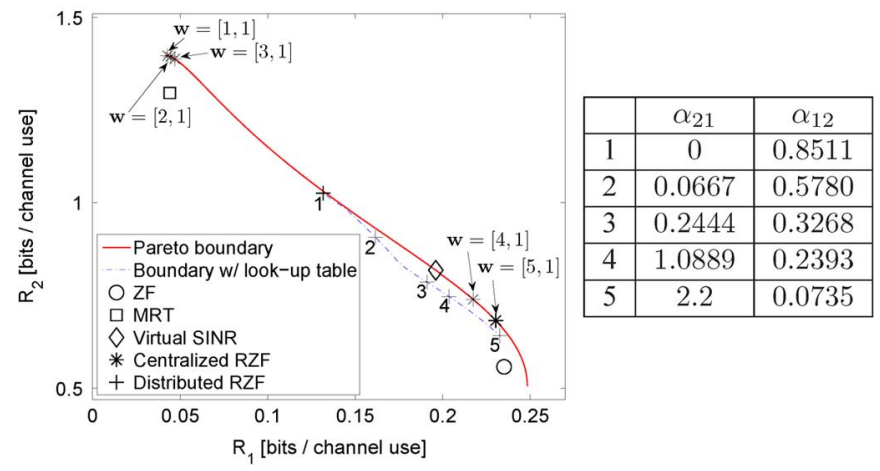

(b)

Fig. 6. Performance of RZFCB with the proposed rate control algorithms: The centralized rate control, marked with $*$, searches for the weighted sum rate maximizing point. (The weight vector $\mathbf{w}$ is shown in the figure.) The distributed rate control scheme, marked with + , sets the interference leakage levels as shown in the table. In Figs. 6(a) and (b), 'virtual SINR' denotes the rate-tuple obtained by the virtual SINR (or SLNR) beamforming method in [15]. The true Pareto boundary was found by using the result in [30].

the utility function is upper bounded and the proposed algorithm yields a monotonically increasing sequence of utility function values. Furthermore, the proposed algorithm is also stable by the monotone convergence theorem. Fig. 5 shows the convergence behavior of the proposed utility function based algorithm for 10 different channel realizations when $K=N=3, P_{i}=10$, $\sigma_{i}=10(i=1,2,3)$, and $u\left(\left\{R_{i}\right\}\right)=\sum_{i} R_{i} / 3$. It is shown in the figure that the proposed algorithm converges in a few iterations. Figs. 6(a) and (b) show the rate-tuple performance in the case of two users. It is seen that the proposed centralized approach almost achieves the Pareto boundary. However, as mentioned already, the convergence of the proposed algorithm to the global optimum is not guaranteed.

\section{B. A Distributed Heuristic Approach and Practical Considerations}

The proposed centralized algorithm in the previous subsection requires central processing with the knowledge of all $\left\{\mathbf{h}_{j i}\right.$ : $i, j=1,2, \ldots, K\}$ and $\left\{\sigma_{j}^{2}: j=1,2, \ldots, K\right\}$. This reduces the practicality of the centralized approach when communication among the base stations is limited or experiences large delay as in real systems. Note that the RZFCB framework in Problem 2 itself is distributed. Transmitter $i$ only needs to know $\left\{\mathbf{h}_{j i}, j=1,2, \ldots, K\right\}$ and $\left\{\sigma_{j}^{2}, j=1,2, \ldots, K\right\}$ and needs to control $\left\{\alpha_{1 i}, \ldots, \alpha_{i-1, i}, \alpha_{i+1, i}, \alpha_{K i}\right\}$. In the RZF framework, heuristic rate control is possible with the knowledge of $\left\{\mathbf{h}_{j i}, j=1,2, \ldots, K\right\}$ and $\left\{\sigma_{j}^{2}, j=1,2, \ldots, K\right\}$ at transmitter $i$. For fully distributed CB operation with limited inter-base station communication, instantaneous information such as the channel vectors should not be exchanged since inter-base station communication delay is typically larger than the channel coherence time for mobile users. One possible way to roughly control the rate-tuple in the network is to design a table composed of sets of interference relaxation parameters, as in the right side of Fig. 6, based on the channel statistics. When the transmitters form a coordinating cluster, they can negotiate their rates based on the requests from their receivers for a communication session. In this phase, one set of interference relaxation levels from the table is picked, shared among the base stations, and used during the communication session.

Heuristic guidelines to design the parameter table are based on the RZF parameterization itself. Note that $\epsilon_{i}=\sum_{j \neq i} \alpha_{i j}$ in (6) is the additional interference power relative to thermal noise power $\sigma_{i}^{2}$ at receiver $i$ and $\epsilon_{i}=1$ means that the SINR of receiver $i$ is lower than the SNR of the same receiver by $3 \mathrm{~dB}$. Thus, the designed interference level should not be too high compared to the thermal noise level. Furthermore, to (roughly) obtain corner points of the Pareto boundary of the rate region, another heuristic idea works. One transmitter should use a nearly MF beam vector, and the rest of the transmitters should use nearly ZF beam vectors. More systematic ways based on vast computer simulation can be considered to design the parameter table. One possible way is as follows. We first generate a set of channel vectors randomly according to the channel statistics. For this realized channel set, we obtain graphs of interference relaxation parameters on the Pareto boundary. The process is repeated over many different channel realizations and the best fitting graphs are obtained from the graphs of interference relaxation parameters of different channel realizations by some regression model. Finally, the table is constructed by selecting some points in the best fitting graphs. The parameter table in the right side of Fig. 6 is obtained in this manner for $K=N=2$ when each element of channel vector is i.i.d. zero-mean complex Gaussian distributed with unit variance and the SNR is $0 \mathrm{~dB}$. Figs. 6(a) and (b) show the rate control performance of the parameter table designed in this manner for two different channel realizations. It is seen that the heuristic method performs well; the five rate points are all near the Pareto boundary for each figure. 
Several advantages in the RZFCB are summarized below.

- Real-time fully distributed operation is possible based on the proposed heuristic control approach. Transmitter $i$ only needs to know $\left\{\mathbf{h}_{j i}: j=1,2, \ldots, K\right\}$ and $\left\{\sigma_{j}^{2}: j=\right.$ $1,2, \ldots, K\}$.

- Once transmitter $i$ knows $\left\{\alpha_{1 i}, \ldots, \alpha_{i-1, i}, \alpha_{i+1, i}, \alpha_{K i}\right\}$, there exists a very fast algorithm, the SOPC algorithm, to design the RZFCB beam vector. Furthermore, in the case of $K=3$, there is an approximate closed-form solution.

- Transmitter $i$ knows its SINR and achievable rate exactly, and its achievable rate is given by $R_{i}=\log \left(1+\frac{\left|\mathbf{h}_{i i}^{H} \mathbf{v}_{i}\right|^{2}}{\left(1+\epsilon_{i}\right) \sigma_{i}^{2}}\right)$. So, transmission based on this rate will be successful with high probability. This is true even when $\left\{\alpha_{j i}\right\}$ are designed suboptimally, i.e., away from the Pareto boundary of the rate region. Thus, the RZFCB scheme is robust.

- On the contrary to the ZF scheme, RZFCB does not require $N \geq K$.

\section{RZFCB FOR MIMO INTERFERENCE CHANNELS}

In this section, we consider the case that both transmitters and receivers are equipped with multiple antennas i.e., MIMO interference channels. In the MIMO case, we consider the weighted sum rate maximization under the RZF framework and then propose a solution to the MIMO RZFCB based on the projected gradient method [19]. The rate control idea in the MISO case can be applied to the MIMO case too.

\section{A. Problem Formulation}

We assume that each receiver has $M$ receive antennas and each transmitter has $N$ transmit antennas. In this case, the received signal at receiver $i$ is given by

$$
\mathbf{y}_{i}=\mathbf{H}_{i i} \mathbf{V}_{i} \mathbf{s}_{i}+\sum_{j \neq i} \mathbf{H}_{i j} \mathbf{V}_{j} \mathbf{s}_{j}+\mathbf{n}_{i}
$$

where $\mathbf{H}_{i j}$ is the $M \times N$ channel matrix from transmitter $j$ to receiver $i, \mathbf{V}_{i}$ is the $N \times d_{i}$ beamforming matrix, $\mathbf{s}_{i}$ is the $d_{i} \times 1$ transmit symbol vector at transmitter $i$ from a Gaussian codebook with $\mathbf{s}_{i} \sim \mathcal{C N}\left(0, \mathbf{I}_{d_{i}}\right)$, and $\mathbf{n}_{i} \sim \mathcal{C N}\left(0, \sigma_{i}^{2} \mathbf{I}\right)$ is the additive noise. As in the MISO case, we have a transmit power constraint, $\left\|\mathbf{V}_{j}\right\|_{F}^{2} \leq P_{j}$, for transmitter $j$. The proposed RZF constraint in the MIMO case is given by an inequality with the Frobenius norm as

$$
\left\|\mathbf{H}_{j i} \mathbf{V}_{i}\right\|_{F}^{2} \leq \alpha_{j i} \sigma_{j}^{2}, \quad \forall i, j \neq i
$$

for some constant $\alpha_{j i} \geq 0$. As in the MISO case, the RZF constraints reduce to ZF constraints when $\alpha_{j i}=0$ for all $i, j \neq$ $i$. With the MIMO RZF constraints, a cooperative beam design problem that maximizes the weighted sum rate is formulated as follows:

Problem 4 (RZF Cooperative Beamforming Problem):

$$
\underset{\left\{\mathbf{V}_{i}\right\}}{\operatorname{maximize}} \sum_{i=1}^{K} w_{i} \log \left|\mathbf{I}_{M}+\left(\sigma_{i}^{2} \mathbf{I}+\mathbf{B}_{i}\right)^{-1} \mathbf{H}_{i i} \mathbf{V}_{i} \mathbf{V}_{i}^{H} \mathbf{H}_{i i}^{H}\right| \text {, }
$$

subject to $\left\|\mathbf{H}_{j i} \mathbf{V}_{i}\right\|_{F}^{2} \leq \alpha_{j i} \sigma_{j}^{2}, \quad \forall i, j \neq i$,

$$
\left\|\mathbf{V}_{i}\right\|_{F}^{2} \leq P_{i}, \quad \forall i
$$

where $w_{i} \geq 0, \sum_{i} w_{i}=1$, and $\mathbf{B}_{i}=\sum_{j \neq i} \mathbf{H}_{i j} \mathbf{V}_{j} \mathbf{V}_{j}^{H} \mathbf{H}_{i j}^{H}$ is the interference covariance matrix at receiver $i$.

Note that, in Problem 4, the interference from other transmitters is incorporated in the rate formula through the interference covariance matrix $\mathbf{B}_{i}$ capturing the residual inter-cell interference under the RZF constraints. As in the MISO case, we will derive a lower bound on the rate of each user by exploiting the RZF constraints to convert the joint design problem into a set of separate design problems. Note that, under the RZF constraints, the total power of interference from undesired transmitters is upper bounded as

$$
\operatorname{tr}\left(\mathbf{B}_{i}\right)=\sum_{j \neq i}\left\|\mathbf{H}_{i j} \mathbf{V}_{j}\right\|_{F}^{2} \leq \sigma_{i}^{2} \sum_{j \neq i} \alpha_{i j}=: \epsilon_{i} \sigma_{i}^{2}
$$

which implies $\mathbf{B}_{i} \leq \epsilon_{i} \sigma_{i}^{2} \mathbf{I}$.

Hassibi and Hochwald derived a lower bound on the ergodic rate of a MIMO channel with interference [32]. However, their result is not directly applicable here since the rate here is for an instantaneous channel realization. Thus, we present a new lower bound under the RZF interference constraints in the following Lemma.

Lemma 3: A lower bound on the rate of receiver $i$ under the RZF constraints is given by

$$
\begin{aligned}
\log \left|\mathbf{I}_{M}+\left(\sigma_{i}^{2} \mathbf{I}+\mathbf{B}_{i}\right)^{-1} \mathbf{H}_{i i} \mathbf{V}_{i} \mathbf{V}_{i}^{H} \mathbf{H}_{i i}^{H}\right| \\
\geq \log \left|\mathbf{I}_{M}+\frac{1}{\sigma_{i}^{2}\left(1+\epsilon_{i}\right)} \mathbf{H}_{i i} \mathbf{V}_{i} \mathbf{V}_{i}^{H} \mathbf{H}_{i i}^{H}\right|
\end{aligned}
$$

where $\operatorname{tr}\left(\mathbf{B}_{i}\right)=\sum_{j \neq i}\left\|\mathbf{H}_{i j} \mathbf{V}_{j}\right\|_{F}^{2} \leq \epsilon_{i} \sigma_{i}^{2}$ for all $i$.

Proof: The rate at receiver $i$ is given by

$$
\log \left|\mathbf{I}+\boldsymbol{\Phi}_{i}^{-1} \mathbf{A}_{i}\right|=\log \prod_{k=1}^{M}\left(1+\lambda_{k}\left(\boldsymbol{\Phi}_{i}^{-1} \mathbf{A}_{i}\right)\right)
$$

where $\boldsymbol{\Phi}_{i}=\sigma_{i}^{2} \mathbf{I}+\mathbf{B}_{i}, \mathbf{A}_{i}=\mathbf{H}_{i i} \mathbf{V}_{i} \mathbf{V}_{i}^{H} \mathbf{H}_{i i}^{H}$, and $\lambda_{k}(\mathbf{X})$ denotes the $k$-th largest eigenvalue of $\mathbf{X}$. By the Rayleigh-Ritz theorem ([33], p. 176), we have

$$
\begin{aligned}
\lambda_{M}\left(\boldsymbol{\Phi}_{i}^{-1} \mathbf{A}_{i}\right) & \leq \frac{\mathbf{x}^{H} \mathbf{A}_{i} \mathbf{x}}{\mathbf{x}^{H} \boldsymbol{\Phi}_{i} \mathbf{x}}=\frac{\mathbf{p}^{H} \boldsymbol{\Phi}_{i}^{-\frac{1}{2}} \mathbf{A}_{i} \boldsymbol{\Phi}_{i}^{-\frac{H}{2}} \mathbf{p}}{\mathbf{p}^{H} \mathbf{p}} \\
& \leq \lambda_{1}\left(\boldsymbol{\Phi}_{i}^{-1} \mathbf{A}_{i}\right)
\end{aligned}
$$

for any non-zero vector $\mathbf{x} \in \mathbb{C}^{M}$ and $\mathbf{p}:=\mathbf{\Phi}_{i}{ }^{\frac{H}{2}} \mathbf{x}$. From the Courant-Fischer theorem ([33], p. 179), the $k$-th largest generalized eigenvalue of $\boldsymbol{\Phi}_{i}^{-1} \mathbf{A}_{i}, k=1, \ldots, M$ is given by

$$
\lambda_{k}\left(\boldsymbol{\Phi}_{i}^{-1} \mathbf{A}_{i}\right)=\max _{\substack{\mathbf{p} \neq 0, \mathbf{p} \in \mathbb{C}^{M}, \mathbf{p} \perp \mathbf{p}_{1}, \ldots, \mathbf{p}_{k-1}}} \frac{\mathbf{p}^{H} \boldsymbol{\Phi}_{i}^{-\frac{1}{2}} \mathbf{A}_{i} \boldsymbol{\Phi}_{i}^{-\frac{H}{2}} \mathbf{p}}{\mathbf{p}^{H} \mathbf{p}}
$$

where $\mathbf{p}_{i}$ is the eigenvector associated with the $i$-th largest eigenvalue of $\boldsymbol{\Phi}_{i}^{-\frac{1}{2}} \mathbf{A}_{i} \boldsymbol{\Phi}_{i}^{-\frac{H}{2}}$. Let 
$\mathbf{A}_{i}=\mathbf{U}_{i} \boldsymbol{\Sigma}_{i} \mathbf{U}_{i}^{H}$ be the eigen-decomposition of $\mathbf{A}_{i}$, where $\boldsymbol{\Sigma}_{i}=\operatorname{diag}\left(\lambda_{1}\left(\mathbf{A}_{i}\right), \ldots, \lambda_{M}\left(\mathbf{A}_{i}\right)\right)$. Then, for all $k$

$$
\begin{aligned}
& \lambda_{k}\left(\boldsymbol{\Phi}_{i}^{-1} \mathbf{A}_{i}\right) \\
& =\max _{\substack{\mathbf{p} \neq 0, \mathbf{p} \in \mathbb{C}^{M}, \mathbf{p} \perp \mathbf{p}_{1}, \ldots, \mathbf{p}_{k-1}}} \frac{\mathbf{p}^{H} \boldsymbol{\Phi}_{i}^{-\frac{1}{2}} \mathbf{A}_{i} \boldsymbol{\Phi}_{i}^{-\frac{H}{2}} \mathbf{p}}{\mathbf{p}^{H} \mathbf{p}}, \\
& =\max _{\substack{\mathbf{p} \neq 0, \mathbf{p} \in \mathbb{C}^{M}, \mathbf{p} \perp \mathbf{p}_{1}, \ldots, \mathbf{p}_{k-1}}} \frac{\mathbf{p}^{H} \boldsymbol{\Phi}_{i}^{-\frac{1}{2}} \mathbf{U}_{i} \boldsymbol{\Sigma}_{i} \mathbf{U}_{i}^{H} \boldsymbol{\Phi}_{i}^{-\frac{H}{2}} \mathbf{p}}{\mathbf{p}^{H} \mathbf{p}}, \\
& =\max _{\substack{\mathbf{z} \neq 0, \mathbf{z} \in \mathbb{C}^{M}, \mathbf{\Phi}_{i}^{H / 2} \mathbf{U}_{i} \mathbf{z} \perp \mathbf{p}_{1}, \ldots, \mathbf{p}_{k-1}}} \frac{\mathbf{z}^{H} \boldsymbol{\Sigma}_{i} \mathbf{z}}{\mathbf{z}^{H} \mathbf{U}_{i}^{H} \mathbf{\Phi}_{i} \mathbf{U}_{i} \mathbf{z}}, \\
& \left(\mathbf{z}:=\mathbf{U}_{i}^{H} \boldsymbol{\Phi}_{i}^{-\frac{H}{2}} \mathbf{p}\right),
\end{aligned}
$$

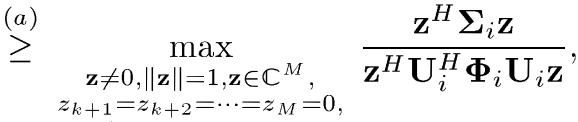

$$
\begin{aligned}
& \boldsymbol{\Phi}_{i}^{H / 2} \mathbf{U}_{i} \mathbf{z} \perp \mathbf{p}_{1}, \ldots, \mathbf{p}_{k-1}, \\
& \left(\mathbf{z}=\left[z_{1}, z_{2}, \ldots, z_{M}\right]^{T}\right)
\end{aligned}
$$

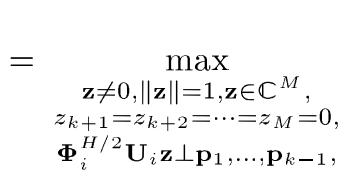

$$
\begin{aligned}
& \frac{\sum_{j=1}^{k} \lambda_{j}\left(\mathbf{A}_{i}\right)\left|z_{j}\right|^{2}}{\mathbf{z}^{H} \mathbf{U}_{i}^{H} \mathbf{\Phi}_{i} \mathbf{U}_{i} \mathbf{z}} \\
& \stackrel{(b)}{\geq} \frac{\lambda_{k}\left(\mathbf{A}_{i}\right)}{\lambda_{1}\left(\boldsymbol{\Phi}_{i}\right)} \text {, }
\end{aligned}
$$

where (a) is satisfied since the feasible set for $\mathbf{z}$ is reduced and (b) is satisfied since $\|\mathbf{z}\|^{2}=\left|z_{1}\right|^{2}+\cdots+\left|z_{k}\right|^{2}=1, \lambda_{1}\left(\mathbf{A}_{i}\right) \geq$ $\cdots \geq \lambda_{k}\left(\mathbf{A}_{i}\right)$, and $\mathbf{z}^{H} \mathbf{U}_{i}^{H} \boldsymbol{\Phi}_{i} \mathbf{U}_{i} \mathbf{z} \leq \lambda_{1}\left(\boldsymbol{\Phi}_{i}\right)$ by Rayleigh-Ritz theorem. Based on (38), a lower bound on the rate is given by

$$
\log \left|\mathbf{I}+\mathbf{\Phi}_{i}^{-1} \mathbf{A}_{i}\right| \geq\left(1+\frac{\lambda_{k}\left(\mathbf{A}_{i}\right)}{\lambda_{1}\left(\mathbf{\Phi}_{i}\right)}\right) .
$$

Since $\boldsymbol{\Phi}_{i}=\sigma_{i}^{2} \mathbf{I}+\sum_{j \neq i} \mathbf{H}_{i j} \mathbf{V}_{j} \mathbf{V}_{j}^{H} \mathbf{H}_{i j}^{H}$, we have $\lambda_{1}\left(\boldsymbol{\Phi}_{i}\right)=$ $\sigma_{i}^{2}+\lambda_{1}\left(\sum_{j \neq i} \mathbf{H}_{i j} \mathbf{V}_{j} \mathbf{V}_{j}^{H} \mathbf{H}_{i j}^{H}\right)$, where the maximum eigenvalue of the interference covariance matrix is upper bounded by $\lambda_{1}\left(\sum_{j \neq i} \mathbf{H}_{i j} \mathbf{V}_{j} \mathbf{V}_{j}^{H} \mathbf{H}_{i j}^{H}\right) \leq \operatorname{tr}\left(\sum_{j \neq i} \mathbf{H}_{i j} \mathbf{V}_{j} \mathbf{V}_{j}^{H} \mathbf{H}_{i j}^{H}\right)=$ $\sum_{j \neq i} \alpha_{i j} \sigma_{i}^{2}=\epsilon_{i} \sigma_{i}^{2}$. Thus, a lower bound of rate at receiver $i$ is given by

$$
\left|\mathbf{I}+\boldsymbol{\Phi}_{i}^{-1} \mathbf{A}_{i}\right| \geq\left|\mathbf{I}_{M}+\frac{1}{\sigma_{i}^{2}\left(1+\epsilon_{i}\right)} \mathbf{A}_{i}\right| .
$$

Note that in (33) the inter-user dependency is removed and the beam design can be performed at each transmitter in a distributed manner. Based on the lower bound (33), the RZFCB problem is now formulated as a distributed problem:

Problem 5 (The MIMO RZFCB Problem):

$$
\begin{array}{cl}
\underset{\mathbf{V}_{i}}{\operatorname{maximize}} & \phi_{i}\left(\mathbf{V}_{i}\right) \\
& :=\log \left|\mathbf{I}_{M}+\frac{1}{\sigma_{i}^{2}\left(1+\epsilon_{i}\right)} \mathbf{H}_{i i} \mathbf{V}_{i} \mathbf{V}_{i}^{H} \mathbf{H}_{i i}^{H}\right|, \\
\text { subject to } & \left\|\mathbf{H}_{j i} \mathbf{V}_{i}\right\|_{F}^{2} \leq \alpha_{j i} \sigma_{j}^{2}, \quad \forall j \neq i \\
& \left\|\mathbf{V}_{i}\right\|_{F}^{2} \leq P_{i},
\end{array}
$$

TABLE III

BeAm Design Algorithm For MIMO IC Using PGM
For each transmitter $i \in\{1, \cdots, K\}$,

0 . Initialize $\mathbf{V}_{i}$ as the $\mathrm{ZF}$ beamforming matrix.

1. Compute gradient of $\phi\left(\mathbf{V}_{i}\right)$.

2. perform the steepest descent shift of $\mathbf{V}_{i}$ :

$\mathbf{V}_{i}= \begin{cases}\mathbf{V}_{i}-\lambda \frac{\phi\left(\mathbf{V}_{i}\right)}{\left\|\nabla \phi\left(\mathbf{V}_{i}\right)\right\|^{2}} \nabla \phi\left(\mathbf{V}_{i}\right), & \text { if } \nabla \phi\left(\mathbf{V}_{i}\right) \neq 0, \\ \mathbf{V}_{i}, & \text { otherwise. }\end{cases}$

Here, $\lambda$ is the step size for the steepest descent.

3. Perform successive metric projections of $\mathbf{V}_{i}$ onto constraint sets.

4. Go to Step 1 and repeat until the relative difference of $\phi_{i}\left(\mathbf{V}_{i}\right)$ is less than a pre-determined threshold.

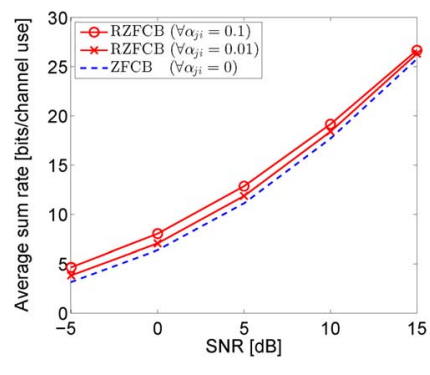

(a)

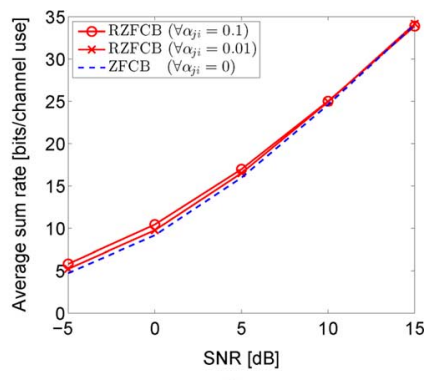

(b)

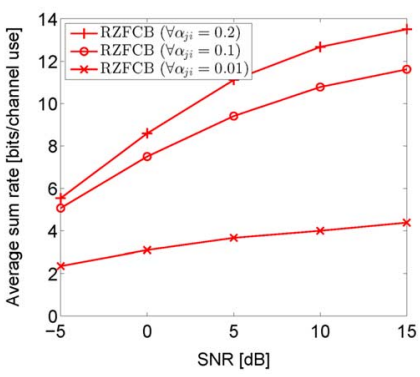

(c)

Fig. 7. Average sum rate of RZFCB: (a) $(K, M, N)=(3,2,6)$, (b) $(K, M, N)=(3,2,8)$, and (c) $(K, M, N)=(4,2,6)$.

for each transmitter $i=1,2, \ldots, K$.

Note that Problem 5 is now fully distributed. One of several known algorithms for constrained optimization can be used to solve Problem 5 for given $\left\{\alpha_{j i}\right\}$. In particular, we choose to use the projected gradient method (PGM) by Goldstein [19]. The proposed PGM-based beam design algorithm for MIMO ICs is provided in Table III. Detailed explanation of the beam design with PGM algorithm is provided in [14].

\section{B. Numerical Results}

In this section, we provide some numerical results for the performance of RZFCB in the MIMO case. We consider three MIMO interference channels with system parameters $(K, M, N)=(3,2,6),(3,2,8)$, and $(4,2,6)$. In each case, we basically set $\alpha_{j i}=0.01$ and 0.1 for all $i$ and $j$. The step size parameter $\lambda$ for the PGM is chosen to be 0.01 for all iterations. For comparison, the performance of ZF beam $\left(\alpha_{j i}=0, \forall i, j\right)$ is provided for $(K, M, N)=(3,2,6)$ and $(3,2,8)$ and the performance of the RZF beam with $\alpha_{j i}=0.2$ is included for $(4$, 2,6). Figs. 7(a), (b), and (c) show the sum rate performance of the ZFCB and RZFCB averaged over 30 independent channel realizations. In Fig. 7(a) it is seen that the RZFCB outperforms 
the ZFCB at all SNR and the gain of the RZFCB over the ZFCB at low SNR is large when $N=K M$. This large gain at low SNR is especially important because most cell-edge receivers operate in the low SNR regime. In Fig. 7(b) it is seen that the ZF scheme performs well when the number of TX antenna is more than enough and the dimension of ZF beams is large, as expected. In the case of $N<K M$ as in Fig. (c), the ZFCB is infeasible but the RZFCB still works well.

\section{CONCLUSION}

We have considered coordinated beamforming for MISO and MIMO interference channels under the RZF framework. In the MISO case, we have shown that the SOPC strategy with a set of well chosen interference relaxation levels is Pareto-optimal. We have provided (approximate) closed-form solutions for the SOPC strategy in the cases of two and three users and the SOPC algorithm in the general case for a given set of interference relaxation levels. In the MIMO case, we have formulated the RZFCB problem as a distributed optimization problem based on a newly derived rate lower bound and have provided an algorithm based on the PGM to solve the MIMO RZFCB beam design problem. We have also considered the rate control problem under the RZFCB framework and have provided a centralized approach and a fully-distributed heuristic approach to control the rate-tuple location roughly along the Pareto boundary of the achievable rate region. Numerical results have validated the RZFCB paradigm.

\section{REFERENCES}

[1] G. Lee, J. Park, Y. Sung, and M. Yukawa, "Coordinated beamforming with relaxed zero forcing," presented at the WCSP, Nanjing, China, Nov. 2011.

[2] Further Advancements for E-UTRA Physical Later Aspects, , V. 3GPP TR 36.814, Mar. 2010.

[3] M. Sawahashi, Y. Kishiyama, A. Morimoto, D. Nishikawa, and M. Tanno, "Coordinated multipoint transmission/reception techniques for LTE-Advanced," IEEE Wireless Commun. Mag., vol. 17, pp. 26-34, Jun. 2010.

[4] E. Jorswieck, E. Larsson, and D. Danev, "Complete characterization of the Pareto boundary for the MISO interference channel," IEEE Trans. Signal Process., vol. 56, pp. 5292-5296, Oct. 2008.

[5] R. Mochaourab and E. Jorswieck, "Optimal beamforming in interference networks with perfect local channel information," IEEE Trans. Signal Process., vol. 59, pp. 1128-1141, Mar. 2011.

[6] J. Lindblom, E. Larsson, and E. Jorswieck, "Parametrization of the MISO IFC rate region: The case of partial channel state information," IEEE Trans. Wireless Commun., vol. 9, pp. 500-504, Feb. 2010.

[7] E. Björnson, R. Zakhour, D. Gesbert, and B. Ottersten, "Cooperate multicell precoding: Rate region characterization and distributed strategies with instantaneous and statistical CSI," IEEE Trans. Signal Process., vol. 58, pp. 4298-4310, Aug. 2010.

[8] J. Qiu, R. Zhang, Z.-Q. Luo, and S. Cui, "Optimal distributed beamforming for MISO interference channels," IEEE Trans. Signal Process., vol. 59, pp. 5638-5643, Nov. 2011.

[9] W. Utschick and J. Brehmer, "Monotonic optimization and framework for coordinated and beamforming in multicell and networks," IEEE Trans. Signal Process., vol. 60, pp. 1899-1909, Apr. 2012.

[10] C. B. Peel, B. M. Hochwald, and A. L. Swindlehurst, "A vector-perturbation technique for near-capacity multiantenna multiuser communication-Part I: Channel inversion and regularization," IEEE Trans. Commun., vol. 53, pp. 195-202, Jan. 2005.

[11] Z. Wang and W. Chen, "Regularized zero-forcing for multiantenna broadcast channels with user selection," IEEE Wireless Commun. Lett., vol. 1, pp. 129-132, Apr. 2012.

[12] S. Wagner, R. Couillet, D. Slock, and M. Debbah, "Asymptotic analysis of regularized zero-forcing precoding in MISO broadcast channels with limited feedback," presented at the ISIT, Austin, TX, USA, Jun. 2010.
[13] M. Sadek, A. Tarighat, and A. H. Sayed, "A leakage-based precoding scheme for downlink multi-user MIMO Channels," IEEE Trans. Wireless Commun., vol. 6, pp. 1711-1721, May 2007.

[14] J. Park, G. Lee, Y. Sung, and M. Yukawa, "Coordinate beamforming with relaxed zero forcing: The sequential orthogonal projection combining method and rate control," Mar. 2012 [Online]. Available: http:// arxiv.org/abs/1203.1758,, ArXiv Pre-Print cs.IT/1203.1758.

[15] R. Zakhour and D. Gesbert, "Coordination on the MISO interference channel using the virtual SINR framework," presented at the WSA 2009, Berlin, Germany, Feb. 2009.

[16] R. Zakhour and D. Gesbert, "Distributed multicell-MISO precoding using the layered virtual SINR framework," IEEE Trans. Wireless Commun., vol. 9, pp. 2444-2448, Aug. 2010.

[17] X. Shang, B. Chen, and H. V. Poor, "Multiuser MISO interference channels with single-user detection: Optimality of beamforming and the achievable rate region," IEEE Trans. Inf. Theory, vol. 57, pp. 4255-4273, Jul. 2011.

[18] R. Zhang and S. Cui, "Cooperative interference management with MISO beamforming," IEEE Trans. Signal Process., vol. 58, pp. 5450-5458, Oct. 2010.

[19] A. A. Goldstein, "Convex programming in Hilbert space," Bull. Amer. Math. Soc., vol. 70, pp. 709-710, 1964.

[20] Q. H. Spencer, A. L. Swindlehurst, and M. Haardt, "Zero-forcing methods for downlink spatial multiplexing in multi-user MIMO channels," IEEE Trans. Signal Process., vol. 52, pp. 461-471, Feb. 2004.

[21] S. Shim, J. S. Kwak, R. W. Heath, Jr., and J. G. Andrews, "Block diagonalization for mult-user MIMO with other-cell interference," IEEE Trans. Wireless Commun., vol. 7, pp. 2671-2681, July 2008.

[22] O. Somekh, O. Simeone, Y. Bar-Ness, A. M. Haimovich, and S. Shamai (Shitz), "Cooperative multicell zero-forcing beamforming in cellular downlink channels," IEEE Trans. Inf. Theory, vol. 55, pp. 3206-3219, Jul. 2009.

[23] E. Larsson and E. Jorswieck, "Competition versus cooperation on the MISO interference channel," IEEE J. Sel. Areas Commun., vol. 26, pp. 1059-1069, Sep. 2008.

[24] M. Á. Vázquez, A. P.-Neira, and M. Á. Lagunas, "A unifying approach to transmit beamforming for the MISO interference channel," in Proc. WSA, Dresden, Germany, Mar. 2012, pp. 152-156.

[25] E. Karipidis, A. Grundinger, J. Lindblom, and E. Larsson, "Paretooptimal beamforming for the MISO interference channel with partial CSI," presented at the CAMSAP, Aruba, Dutch Antilles, Dec. 2009.

[26] J. Lindblom, E. Karipidis, and E. Larsson, "Outage rate regions for the MISO IFC," presented at the Asilomar 2009, Pacific Glove, CA, USA, Nov. 2009.

[27] M. Bengtsson and B. Ottersten, "Optimal downlink beamforming using semidefinite optimization," in Proc. Annu. Allerton Conf. Commun. Control Comput., Sep. 1999, pp. 987-996.

[28] R. G. Bland, D. Goldfarb, and M. J. Todd, "The ellipsoid method: A survey," Oper. Res., vol. 29, pp. 1039-1091, Nov. 1981.

[29] S. Lototsky, Filtering Theory Lecture Notes Univ. of Southern California, Los Angeles, CA, USA, 2011,

[30] R. Mochaourab and E. Jorswieck, "Exchange economy in two-user multiple-input single-output interference channels," IEEE J. Sel. Topics Signal Process., vol. 6, pp. 151-164, Apr. 2012.

[31] E. Jorswieck and E. Larsson, "The MISO interference channel from a game-theoretic perspective: A combination of selfishness and altruism achieves Pareto optimality," in Proc. ICASSP, Las Vegas, NV, USA, Apr. 2008, pp. 2805-2808.

[32] B. Hassibi and M. Hochwald, "How much training is needed in multiple-antenna wireless links?," IEEE Trans. Inf. Theory, vol. 49, pp. 951-963, Apr. 2003.

[33] R. A. Horn and C. R. Johnson, Matrix Analysis. Cambridge, U.K.: Cambridge Univ. Press, 1985.

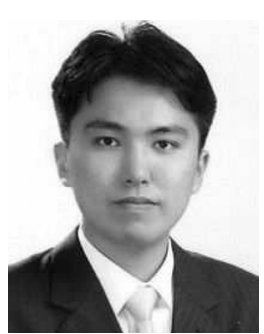

Juho Park (S'06) received the B.S. and M.S. degrees in electrical engineering from KAIST, Daejeon, South Korea, in 2006 and 2008, respectively.

$\mathrm{He}$ is currently working towards the Ph.D. degree in the Department of Electrical Engineering, KAIST. His research interests include signal processing for communications and next generation communication systems. 


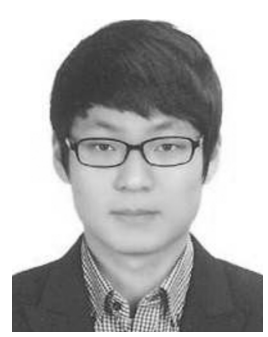

Gilwon Lee (S'10) received the B.S. and M.S. degrees in electrical engineering from KAIST, Daejeon, South Korea, in 2010 and 2012, respectively.

He is currently with the wireless information systems research group, KAIST, working towards the $\mathrm{Ph} . \mathrm{D}$. degree. His research interests include signal processing for communications and nonlinear adaptive filtering.

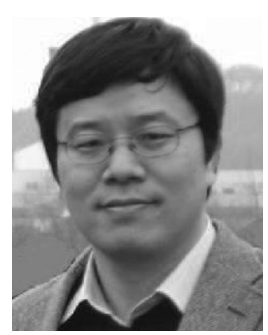

Youngchul Sung (S'92-M'93-SM'09) received the B.S. and M.S. degrees from Seoul National University, Seoul, Korea, in electronics engineering in 1993 and 1995, respectively. After working at LG Electronics, Ltd., Seoul, Korea, from 1995 to 2000, he joined the Ph.D. program in 2000 and received the $\mathrm{Ph} . \mathrm{D}$. degree in electrical and computer engineering from Cornell University, Ithaca, NY, USA, in 2005.

From 2005 until 2007, he was a Senior Engineer in the Corporate R\&D Center of Qualcomm, Inc., San Diego, CA, USA, and participated in design of WCDMA basestation modem. Since 2007, he has been on the faculty of the Department of Electrical Engineering in KAIST, Daejeon, Korea. His research interests include signal processing for communications, statistical signal processing, and asymptotic statistics with applications to next-generation wireless communications and related areas.

Dr. Sung is an associate member of the IEEE Signal Processing Society Signal Processing for Communications and Networking (SPCOM) Technical Committee, an Associate Editor of the IEEE Signal Processing LeTTERS, a member of Signal and Information Processing Theory and Methods (SIPTM) Technical Committee of Asia-Pacific Signal and Information Processing Association (APSIPA), Vice-Chair of IEEE ComSoc Asia-Pacific Board MCC, and
Technical Program Committee member of conferences, including Globecom 2009-2013, ICC 2011, MILCOM 2010, DCOSS 2010, WiOpt 2009, and its sponsorship chair, APSIPA 2009-2012, and IEEE SAM 2008.

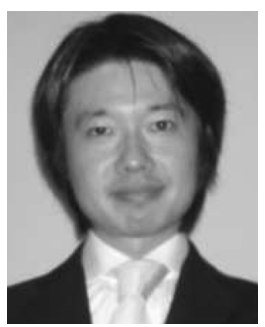

Masahiro Yukawa (M'06) received the B.E., M.E., and Ph.D. degrees from the Tokyo Institute of Technology, Tokyo, Japan, in 2002, 2004, and 2006, respectively.

After studying as a Visiting Researcher at the University of York, U.K., from October 2006 to March 2007, he worked as a Special Postdoctoral Researcher for the Next Generation Mobile Communications Laboratory at RIKEN, Saitama, Japan, from April 2007 to March 2008, and for the Brain Science Institute at RIKEN from April 2008 to March 2010. From August to November 2008, he was a Guest Researcher at the Associate Institute for Signal Processing, the Technical University of Munich, Germany. He was an Associate Professor at the Department of Electrical and Electronic Engineering, Niigata University, Japan, from April 2010 to March 2013. He is currently an Assistant Professor at the Department of Electronics and Electrical Engineering, Keio University, Japan. His current research interests are in mathematical signal processing, nonlinear adaptive filtering, and sparse signal processing.

Dr. Yukawa serves as an Associate Editor for the IEICE Transactions on Fundamentals of Electronics, Communications and Computer Sciences, and the Journal of Multidimensional Systems and Signal Processing, both by Springer. From April 2005 to March 2007, he was a recipient of the Research Fellowship of the Japan Society for the Promotion of Science (JSPS). He received the Excellent Paper Award and the Young Researcher Award from the IEICE in 2006 and in 2010, respectively, the Yasujiro Niwa Outstanding Paper Award from Tokyo Denki University in 2007, and the Ericsson Young Scientist Award from Nippon Ericsson in 2009. He is a member of the Institute of Electrical, Information and Communication Engineers (IEICE) of Japan. 